\title{
Genome analysis of three Pneumocystis species reveals adaptation mechanisms to life exclusively in mammalian hosts
}

Liang Ma ${ }^{1, \star}$, Zehua Chen ${ }^{2, \star}$, Da Wei Huang ${ }^{3, \dagger}$, Geetha Kutty $^{1}$, Mayumi Ishihara ${ }^{4}$, Honghui Wang ${ }^{1}$,

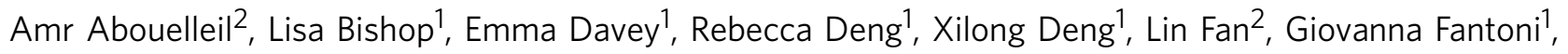
Michael Fitzgerald ${ }^{2}$, Emile Gogineni ${ }^{1}$, Jonathan M. Goldberg ${ }^{2, \dagger}$, Grace Handley ${ }^{1}$, Xiaojun Hu ${ }^{3}$, Charles Huber ${ }^{1}$, Xiaoli Jiao ${ }^{3}$, Kristine Jones ${ }^{3}$, Joshua Z. Levin², Yueqin Liu', Pendexter Macdonald², Alexandre Melnikov², Castle Raley ${ }^{3}$, Monica Sassi ${ }^{1}$, Brad T. Sherman ${ }^{3}$, Xiaohong Song ${ }^{1}$, Sean Sykes ${ }^{2}$, Bao Tran ${ }^{3}$, Laura Walsh ${ }^{1}$, Yun Xia , Jun Yang ${ }^{3}$, Sarah Young ${ }^{2}$, Qiandong Zeng ${ }^{2}$, Xin Zheng ${ }^{3}$, Robert Stephens ${ }^{3}$, Chad Nusbaum², Bruce W. Birren², Parastoo Azadi ${ }^{4}$, Richard A. Lempicki ${ }^{3}$, Christina A. Cuomo ${ }^{2, \star \star} \&$ Joseph A. Kovacs ${ }^{1, \star \star}$

Pneumocystis jirovecii is a major cause of life-threatening pneumonia in immunosuppressed patients including transplant recipients and those with HIV/AIDS, yet surprisingly little is known about the biology of this fungal pathogen. Here we report near complete genome assemblies for three Pneumocystis species that infect humans, rats and mice. Pneumocystis genomes are highly compact relative to other fungi, with substantial reductions of ribosomal RNA genes, transporters, transcription factors and many metabolic pathways, but contain expansions of surface proteins, especially a unique and complex surface glycoprotein superfamily, as well as proteases and RNA processing proteins. Unexpectedly, the key fungal cell wall components chitin and outer chain $\mathrm{N}$-mannans are absent, based on genome content and experimental validation. Our findings suggest that Pneumocystis has developed unique mechanisms of adaptation to life exclusively in mammalian hosts, including dependence on the lungs for gas and nutrients and highly efficient strategies to escape both host innate and acquired immune defenses.

\footnotetext{
${ }^{1}$ Critical Care Medicine Department, NIH Clinical Center, National Institutes of Health, Building 10, Room 2C145, 10 Center Drive, Bethesda, Maryland 20892, USA. ${ }^{2}$ Genome Sequencing and Analysis Program, Broad Institute of Harvard and Massachusetts Institute of Technology, Cambridge, Massachusetts 02142, USA. ${ }^{3}$ Leidos BioMedical Research, Inc., Frederick National Laboratory for Cancer Research, Frederick, Maryland 21701, USA. ${ }^{4}$ Complex Carbohydrate Research Center, University of Georgia, Athens, Georgia 30602, USA. ${ }^{\star}$ These authors contributed equally to this work. ${ }^{\star \star}$ These authors jointly supervised this work. †Present addresses: Laboratory of Molecular Biology, National Cancer Institute, Bethesda, Maryland 20892, USA (D.W.H.); Department of Infectious and Immunological Diseases, Harvard T. H. Chan School of Public Health, Boston, Massachusetts 02115, USA (J.M.G.). Correspondence and requests for materials should be addressed to L.M. (email: liang.ma@nih.gov) or to C.A.C. (email: cuomo@broadinstitute.org) or to J.A.K. (email: jkovacs@nih.gov).
} 
$\mathrm{P}$ neumocystis organisms were first described in 1909, but Pneumocystis became a prominent cause of morbidity and mortality only in the late 20th century, as more humans become susceptible due to HIV/AIDS or immunosuppressive therapies developed for cancer, transplants and inflammatory diseases. The genus Pneumocystis comprises a group of highly diversified microorganisms that reside in the lungs of humans and other mammals. Pneumocystis jirovecii, the species that infects humans, causes life-threatening pneumonia (termed Pneumocystis pneumonia or PCP) exclusively in immunosuppressed patients, and has been responsible for many thousands of deaths over the past 30 years. Despite improvements in diagnosis and treatment, PCP still has an estimated mortality rate of $5-30 \%$. Recently, the frequency of PCP has been increasing in non-HIV immunosuppressed patients, especially renal transplant patients, in whom multiple outbreaks have been reported in the past decade ${ }^{1,2}$. Moreover, colonization with Pneumocystis is being recognized in an expanding population of patients with underlying pulmonary disease, potentially contributing to accelerated deterioration in pulmonary function $^{3}$. While a number of drugs, including trimethoprimsulfamethoxazole and atovaquone, are effective in treating and preventing PCP, there have been rising concerns of drug resistance to the best available therapeutic agents ${ }^{4}$.

Unique among fungal species, Pneumocystis have adapted to and co-evolved with individual mammalian host species such that each Pneumocystis species can infect only a single host species; the basis for this specificity is currently unknown. Pneumocystis has two primary stages: spherical cysts, which have a thick cell wall that is rigid, and the more numerous, pleomorphic (amoeboid) trophic forms, which have a thin, flexible cell wall that is often tightly attached to type I pneumocytes in lung alveoli. Animal studies suggest that the cyst form is responsible for transmission between hosts ${ }^{5}$. Although the life cycle remains undefined, it has been hypothesized that trophic forms can undergo binary fission or, alternatively, conjugate and undergo meiosis, leading to the development of cysts with up to eight intracystic bodies, which can subsequently be released as new trophic forms ${ }^{4}$.

Despite its medical importance, our knowledge of the basic biology of Pneumocystis remains very limited, in large part due to an inability to reproducibly culture the organism in vitro, notwithstanding intense efforts by numerous investigators over the past three decades. Genetic manipulation by transformation is currently untenable. As a consequence, options for developing rational new therapies are currently limited.

To help address these deficiencies, we integrate here wholegenome sequencing and biochemical analysis to gain deeper insights into the biology of $P$. jirovecii (the human pathogen) and two related species infecting mice (Pneumocystis murina) and rats (Pneumocystis carinii) that serve as important models for studying the pathogenesis and treatment of PCP. While efforts to sequence a Pneumocystis genome began two decades ago ${ }^{6}$, the currently available assemblies and annotations of the P. carinii ${ }^{7}$ and $P$. jirovecii ${ }^{8}$ genomes remain largely fragmentary (with 4,278 and 358 contigs, respectively) and incomplete; the $P$. murina genome has not been previously sequenced. Furthermore, accurate assembly has been hampered by the presence of highly repetitive sequences in the Pneumocystis genome, including the very large multi-copy gene families encoding the major surface glycoproteins (Msg) and kexin proteases, which have been almost entirely excluded from the current genome assemblies of both $P$. carinii ${ }^{7}$ and $P$. jirovecii ${ }^{8}$. Analysis of these draft genomes has provided insights into the sexual reproductive system ${ }^{9}$ and several metabolic pathways such as the amino acid biosynthesis, nitrogen and sulfur assimilation, glyoxylate cycle, myo-inositol biosynthesis, thiamine biosynthesis and purine degradation ${ }^{8-13}$. However, the fragmentary and incomplete status of both genomes has prevented a comprehensive and precise analysis of chromosome structure and gene gain and loss.

In the present study, we generate high-quality genome assemblies that approach the chromosomal level for these three species, and perform RNA-Seq to improve predicted gene structures and to examine gene expression during infection. We also utilize long-read sequencing to characterize the msg and kexin gene families, and perform biochemical analysis of the key cell wall components chitin and mannan, to experimentally validate genome predictions. We present the major differences in genome content and functional attributes of Pneumocystis in comparison with other fungi and highlight the unique features of Pneumocystis species, including cell wall modifications and metabolic shifts that suggest new mechanisms of adaptation to growth as obligate pulmonary pathogens.

\section{Results}

Conservation and content of highly reduced genomes. We generated three high-quality genome assemblies, including the first $P$. murina genome assembly and new assemblies for $P$. carinii and $P$. jirovecii, all of which are at or near the chromosome level and contain the complete gene set except for a small number of $m s g$ (in all three species) or kexin genes (in $P$. carinii alone) in several subtelomeric regions that may remain unidentified. Compared with these assemblies, the previously reported assemblies for $P$. carinii ${ }^{7}$ and $P$. jirovecii ${ }^{8}$ are less complete and continuous, with some genes missing entirely or in part, as evidenced by the shorter average gene and protein lengths, the absence of $\sim 30 \%$ of exons in $P$. jirovecii (Supplementary Table 1), and the lack of both $m s g$ and kexin genes in $P$. carinii and $m s g$ genes in $P$. jirovecii.

The $P$. murina genome assembly is $7.5 \mathrm{Mb}$ in size across 17 scaffolds, which range in size from 292 to $588 \mathrm{~kb}$ (Supplementary Fig. 1a), consistent with the number and size of chromosomes revealed by electrophoretic karyotyping and Southern blotting (Supplementary Figs 2 and 3; Supplementary Note 1). The $P$. carinii genome assembly is $7.66 \mathrm{Mb}$ in size across 17 scaffolds, which range in size from 268 to $635 \mathrm{~kb}$ (Supplementary Fig. 1b), in line with previously reported chromosome number and size from electrophoretic karyotyping experiments ${ }^{14}$. The $P$. jirovecii genome assembly is $8.4 \mathrm{Mb}$ in size across 20 scaffolds, ranging in size from 72 to $635 \mathrm{~kb}$ (Supplementary Fig. 1c). Previous studies have estimated the $P$. jirovecii genome to be $7.0 \mathrm{Mb}$ in size distributed over $12-13$ chromosomes ${ }^{15}$, though this estimate may be inaccurate given the poor quality of available $P$. jirovecii samples.

The genomes of $P$. murina and $P$. carinii are very similar in total length, chromosome structure and gene organization, and show limited rearrangements while the genome of $P$. jirovecii is highly rearranged (Fig. 1a; Supplementary Fig. 4; Supplementary Note 2). Between two $P$. jirovecii strains, there is $\sim 0.3 \%$ genetic variation, with subtelomeric regions showing the highest diversity (Fig. 1b) as observed in some other fungi ${ }^{16}$. Despite these rearrangements, conserved syntenic regions include nearly all genes of $P$. murina compared with either $P$. carinii $(96.1 \%)$ or P. jirovecii (92.9\%). In contrast, comparison to other phylogenetically related fungi identified only small blocks of three to seven genes with a conserved order in comparison of P. murina to Schizosaccharomyces pombe $e^{17}(8.1 \%)$ or Taphrina deformans $^{18}(3.3 \%)$. The high number of inter-chromosomal rearrangements in Pneumocystis contrasts to the primarily intra-chromosomal pattern reported for Saccharomycetes ${ }^{19}$ and other ascomycetes. 


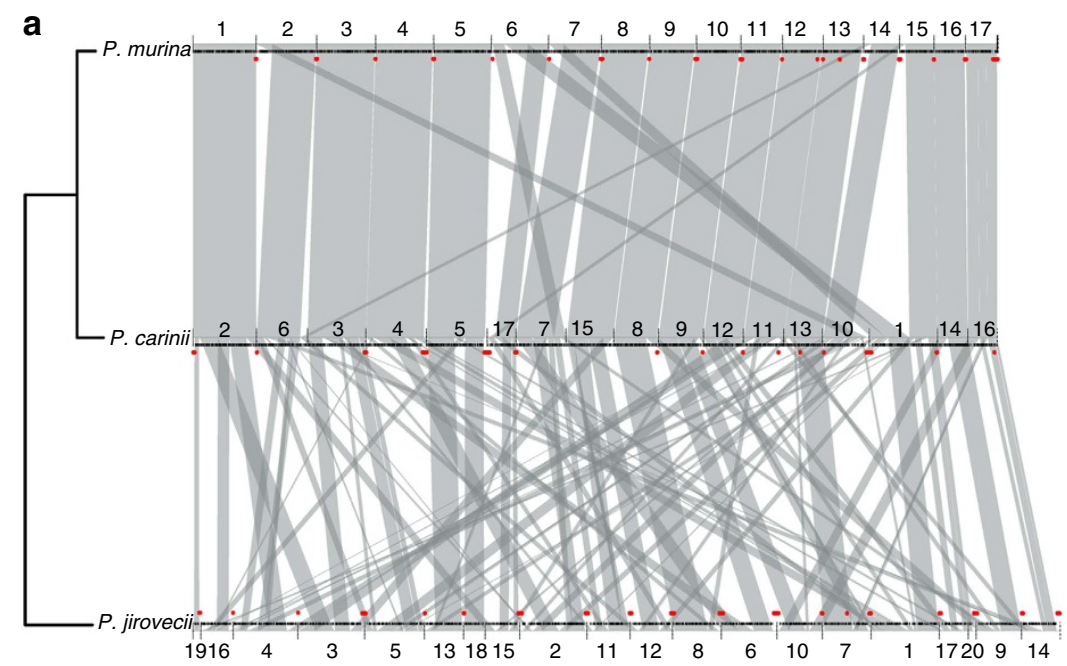

b

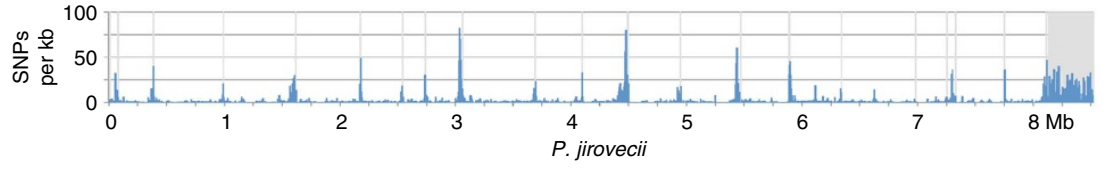

Figure 1 | Conservation of Pneumocystis genome structure. (a) Conserved synteny among three Pneumocystis genomes. Shared syntenic regions are depicted with grey boxes. Scaffold numbers are listed on the $x$-axis, and red dots indicate the location of msg genes. (b) Genome-wide SNP frequency between the P. jirovecii isolates from the United States (RU7) and Switzerland (SE8) ${ }^{8}$ with the same scaffold order as in top panel. The region beyond 8 Mb with a high number of SNPs is composed primarily of small scaffolds containing msg genes not assembled into the 20 large scaffolds. Using our genome assembly (RU7) as a reference, we identified a total of 24,902 SNPs, or 1 every 337 bases, between these 2 isolates, which are over-represented in subtelomeric regions where msg genes are found.

Table 1 | Comparison of Pneumocystis and related fungal genomes.

\begin{tabular}{|c|c|c|c|c|c|c|c|c|}
\hline Species $^{\star}$ & $\begin{array}{l}\text { Chromosomes or } \\
\text { scaffolds (count) }\end{array}$ & $\begin{array}{c}\text { Genome } \\
\text { size }(M b)\end{array}$ & $\begin{array}{l}\text { GC content } \\
(\%)\end{array}$ & $\begin{array}{l}\text { Protein coding } \\
\text { genes (count) }\end{array}$ & $\begin{array}{c}\text { Exons per } \\
\text { gene (count) }\end{array}$ & $\begin{array}{l}\text { tRNA genes } \\
\text { (count) }\end{array}$ & $\begin{array}{l}\text { rRNA genes }{ }^{\dagger} \\
\text { (count) }\end{array}$ & $\begin{array}{l}\text { Intergenic distance } \\
\text { (mean, bp) }\end{array}$ \\
\hline P. murina & 17 & 7.50 & 26.9 & 3623 & 6.08 & 47 & 5 & 423 \\
\hline P. carinii & 17 & 7.66 & 33.2 & 3646 & 5.97 & 45 & 5 & 430 \\
\hline T. deformans & 394 & 13.36 & 49.5 & $4661^{\ddagger}$ & 2.10 & 169 & 5 & 827 \\
\hline S. pombe & 3 & 12.57 & 36.1 & 5155 & 1.99 & 174 & $\sim 450$ & 669 \\
\hline S. cerevisiae & 16 & 12.07 & 38.3 & 5863 & 1.06 & 275 & $\sim 560$ & 515 \\
\hline
\end{tabular}

Compared with other fungi, all three Pneumocystis species have a small genome size and a reduced gene set; between 3,675 and 3,812 genes (including transfer RNA (tRNA) and ribosomal RNA (rRNA) genes) are predicted (Table 1) compared with an average of 5,044 genes in the four related Schizosaccharomyces genomes ${ }^{17}$. Other unusual features of the Pneumocystis genomes are their possession of only a single copy of rRNA genes (Supplementary Fig. 5), a minimal number of tRNA genes and an extremely low GC content, all of which are among the lowest in eukaryotes (Table 1). These features may reflect a slow transcription and translation machinery (Supplementary Note 3), which, together with the loss of many biosynthetic pathways as discussed below, may lead to the slow growth of Pneumocystis organisms as observed in animal models, where doubling times are estimated to be $5-8$ days $^{20}$, as well as to the failure to grow in vitro. The reduced genome size and content likely reflects adaptation to and dependence on human and other mammalian hosts, as has been previously noted for Microsporidia ${ }^{21}$.
Gene family expansions and contractions. To examine the predicted functional impact of genome reduction, we examined gene gain and loss in Pneumocystis relative to seven closely related Ascomycete species (Supplementary Fig. 6) using protein homology and subcellular localization analysis tools (Supplementary Methods). To identify common features of reduced genomes, we also compared these changes in gene content with those in two microsporidial species ${ }^{21}$, both of which are intracellular organisms and have the smallest known genomes in the fungal kingdom (Fig. 2). Based on a phylogenetic tree inferred from 413 single copy core orthologues (Supplementary Methods), the three Pneumocystis species cluster with Taphrina deformans $^{18}$ as a sister group to the Schizosaccharomyces species (Supplementary Fig. 6). As previously recognized based on mitochondrial genome sequences ${ }^{22}, P$. murina and $P$. carinii are more closely related to each other than either is to $P$. jirovecii, although all three species show substantial divergence. 


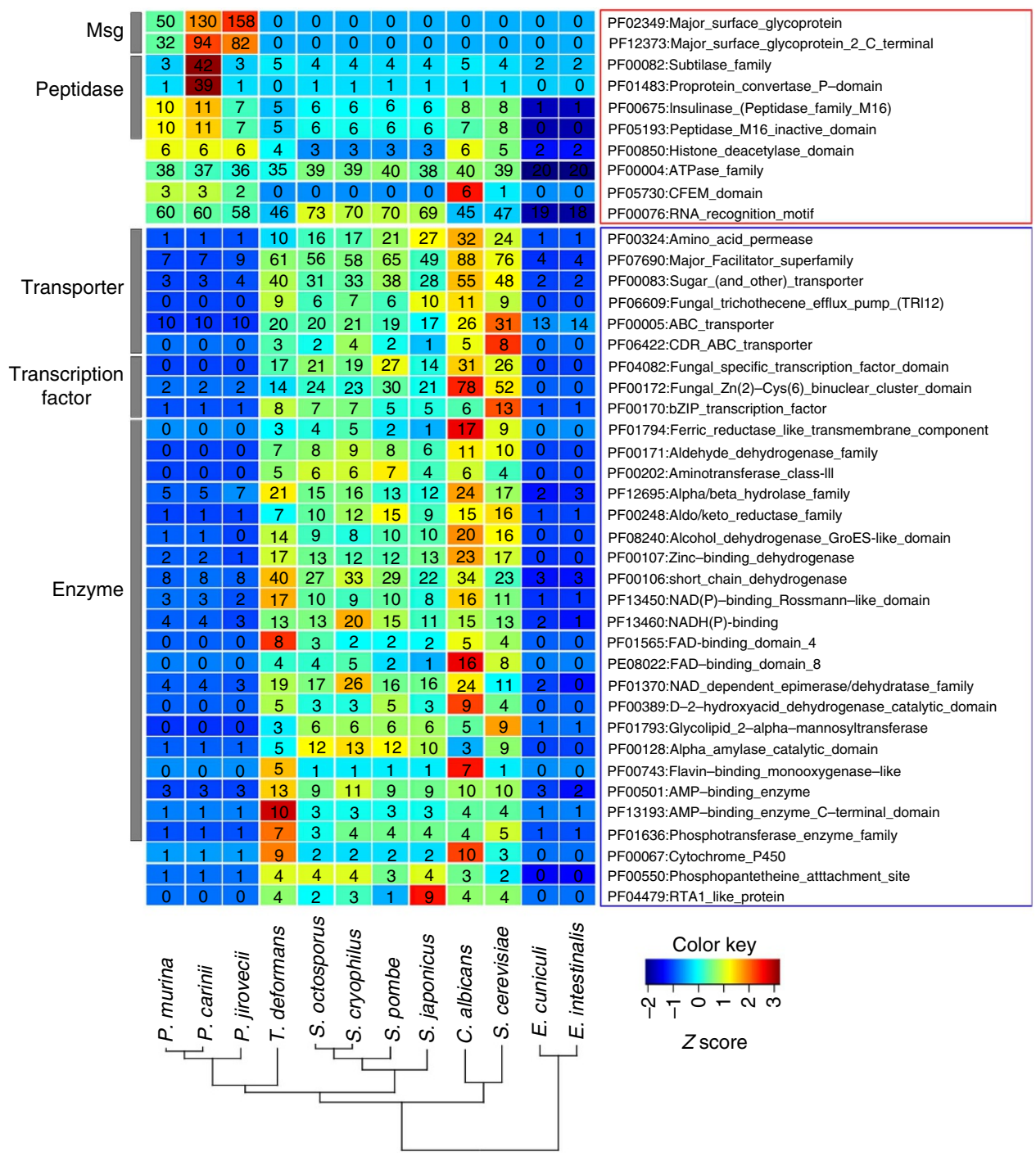

Figure 2 | Protein domains depleted or enriched in Pneumocystis. Significantly enriched (top panel) or depleted (lower panel) Pfam domains (Fisher's exact test, $q$ value $<0.05$ ) are included in the heat map if the domains appear at least twice in the following comparisons: Pneumocystis versus Schizosaccharomyces, Pneumocystis versus Schizosaccharomyces and T. deformans, Pneumocystis versus S. cerevisiae and C. albicans, Pneumocystis versus E. cuniculi and E. intestinalis, Pneumocystis versus all others shown. Broader functional categories of proteins are indicated on the left, while specific Pfam domains are listed on the right. The number of proteins containing each domain is indicated within each box for each species. The heat map is colour coded based on a $Z$ score, as indicated by the key at the bottom right. Fungal species are ordered based on their phylogenetic relationship as indicated at the bottom.

Protein domains enriched in Pneumocystis include hostinteracting cell-surface proteins and proteins required for basic cellular functions. The most significantly enriched domains in Pneumocystis are the Msg domains, which are shared among Pneumocystis species but absent in all other sequenced species. Msg is encoded by a large multi-copy gene superfamily (Fig. 3; Supplementary Table 3; Supplementary Data 1; Supplementary Note 4). Msg genes account for about $3-6 \%$ of an otherwise highly reduced genome, suggesting that the encoded proteins play an essential role in the organism's survival. We found extraordinary diversity in genes encoding the Msg superfamily; there were 64 to 179 unique genes per species, which represent the largest surface protein family identified to date in the fungal kingdom ${ }^{23}$ and which show conservation among most Msg families or subfamilies across species, but also species-specific amplifications. Based on domain structure and phylogenetic analysis, the Msg superfamily is classified into five families, designated as Msg- A, - B, -C, -D and -E families (Fig. 3). The high diversity of these Msg families therefore could specify different proteins at the cell surface, both between strains and between species. Msg is thought to play an important role in hostpathogen interactions and potentially facilitates evasion of host immune responses through antigenic variation ${ }^{23,24}$. The fact that different $P$. jirovecii strains have unique $m s g$ repertoires ${ }^{2,25}$ dramatically expands the potential for antigenic variation, possibly against $\mathrm{T}$-cell rather than B-cell host responses ${ }^{26}$. Msg antigenic variation by gene recombination is supported by the presence of homologoues to all the key genes involved in homologous recombination in Saccharomyces cerevisiae (Supplementary Data 2).

Peptidases of the S8 and M16 families are also highly enriched (Fig. 2; Supplementary Data 3; Supplementary Fig. 7). A large difference between the Pneumocystis species is the expansion of the S8B peptidase subfamily (kexin) in $P$. carinii, which contains 39 copies compared with $0-1$ copy in $P$. murina, $P$. jirovecii and the other analysed fungi. Kexin is potentially involved in the 
a

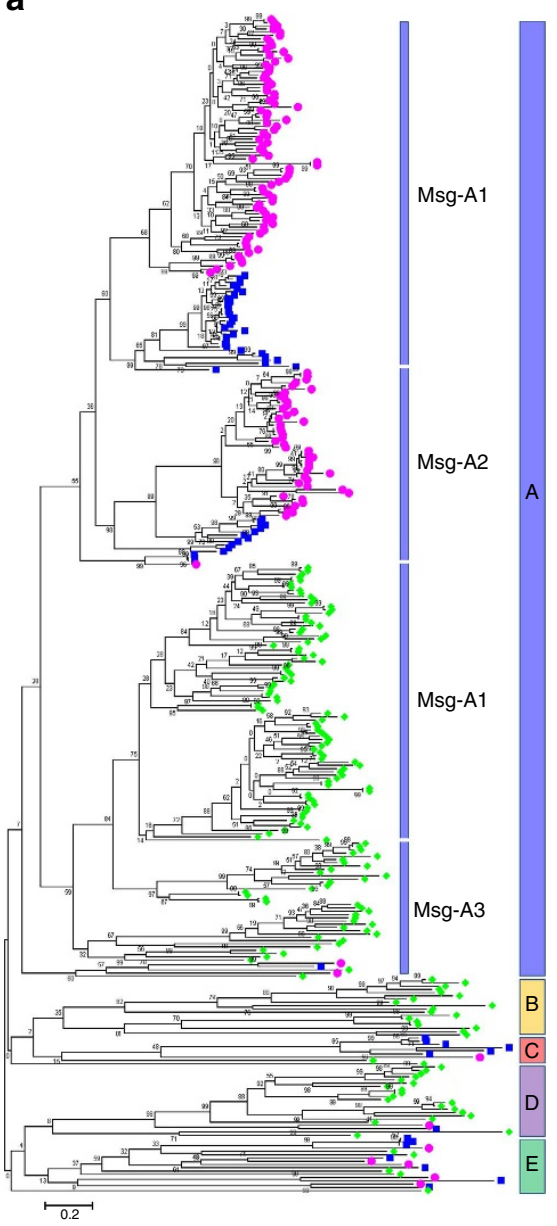

b

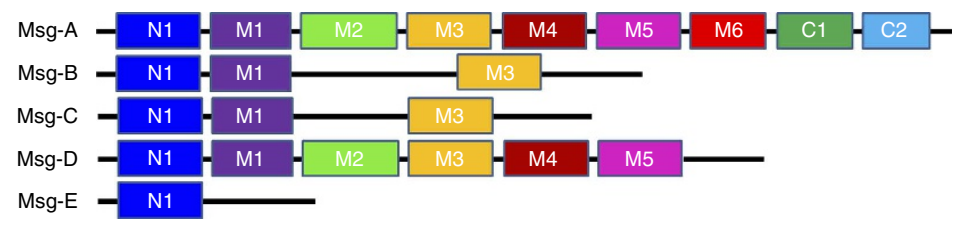

C

M1

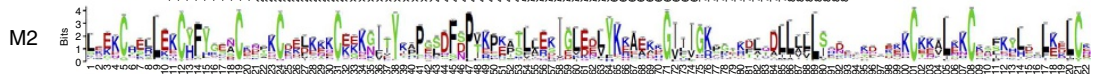

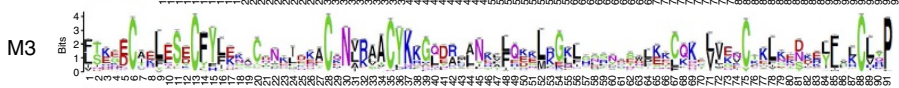

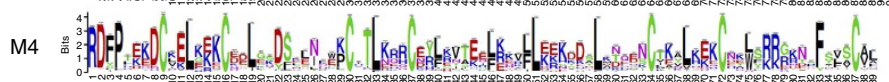

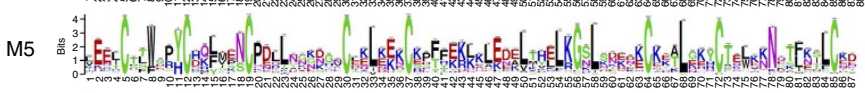

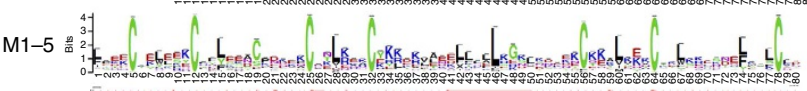

Pfam

MSG

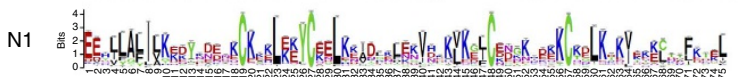

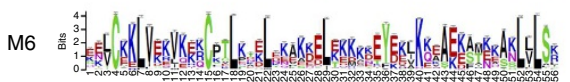

C1

Pfam

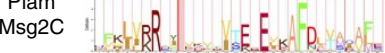

C2

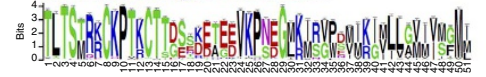

Figure 3 | The Msg superfamily in three Pneumocystis species. (a) Phylogeny of 384 Msg proteins identified in P. murina (blue squares), P. carinii (pink circles) and $P$. jirovecii (green diamonds). They are classified into five families of Msg- $A,-B,-C,-D$ and $-E$, as indicated by the vertical bars on the right side. The Msg-A family is further classified into three subfamilies of Msg-A1 (classical Msg genes), Msg-A2 (Msr genes) and Msg-A3 (other Msg-associated genes). (b) Schematic representations of conserved domains in five Msg families. (c) Sequence logos showing the frequency of amino acid composition in Msg domains. Previously identified Pfam MSG and Pfam Msg2_C domains are included for comparison. Additional information on the Msg domain analysis is provided in Supplementary Note 4.

processing of Msg proteins in the Golgi ${ }^{27-29}$, though in P. carinii many of the kexin genes encode proteins predicted to be on the cell surface through a glycosylphosphatidylinositol anchor ${ }^{29}$ (Supplementary Fig. 7b). The protein encoded by the singlecopy kexin gene in both P. murina and P. jirovecii has been predicted to localize to the Golgi apparatus 27,28 . Other enriched domains include another cell-surface family, the cysteine-rich CFEM (common in fungal extracellular membrane) domain (Supplementary Fig. 8), as well as proteins involved in regulation of transcription, translation and other cellular activities (histone deacetylase family, ATPase family and the RNA recognition motif (RRM), Supplementary Data 4-6), all of which potentially facilitate the survival of Pneumocystis organisms in the host as discussed below.

By contrast, all three Pneumocystis species show extensive reduction of multiple gene families, as expected from their small genome size (Fig. 2). The most significantly reduced Pfam domains in Pneumocystis comprise the following three major categories: (1) transporters, with $<25$ transporters in each Pneumocystis species compared with 131-217 in other ascomycetes for the 6 depleted Pfam domains; (2) transcription factors, with only 3 genes in each Pneumocystis species in the 3 depleted Pfam domains, an order of magnitude fewer than other fungi except for Microsporidia; (3) enzymes, including oxidoreductases, hydrolases, transferases and coenzymes. Most of the significantly depleted domains in Pneumocystis are also depleted in Microsporidia (Fig. 2), suggesting common dependencies of these obligate pathogens on their hosts to complement these biologic functions. While additional transcription factor and transporter-associated domains are conserved (Supplementary Data 7 and 8; Supplementary Fig. 9), the total number of transcription factors and transporters in Pneumocystis is among the lowest in fungi.

Substantial reduction and unique features of metabolic pathways. Since the chromosome-level genome assemblies we generated are more complete than previously reported assemblies 7,8 , we mapped all major metabolic pathways for all three Pneumocystis species (Figs 4 and 5; Supplementary Figs 10 and 11; Supplementary Data 9-19; Supplementary Note 5). Among the most significantly reduced pathways are those involved in amino acid metabolism. As previously noted ${ }^{8,12}$, each Pneumocystis species lacks $\sim 80 \%$ of genes involved in de novo amino acid synthesis in yeast (Supplementary Data 9). In addition, Pneumocystis has impaired capacity for assimilation of inorganic nitrogen and sulfur ${ }^{10}$. Consequently, none of the 20 standard 
amino acids can be synthesized de novo although a few can be synthesized from others. Moreover, in contrast to these earlier reports, we have identified only 1 potential amino acid transporter (Ptr2), which is predicted to localize to the plasma membrane in each species compared with over 20 such transporters in yeasts. Nevertheless, intracellular transport appears highly conserved; nearly half of the 26 mitochondrionand vacuole-associated amino acid transporters in yeast are preserved. While it is believed that polyamines are ubiquitous in all organisms and serve diverse functions, none of the three Pneumocystis genomes encodes any of the enzymes necessary for de novo synthesis of polyamines, though there is one potential polyamine transporter in each species, consistent with previous in vitro studies of $P$. carinii ${ }^{30}$. All three Pneumocystis species have retained the genes required for de novo nucleotide synthesis but are missing nearly all the genes for nucleotide salvage pathways (Supplementary Data 10).

For carbohydrate metabolism, loss of a subset of pathways further highlights nutritional dependency. All three Pneumocystis species have a full complement of genes necessary for uptake and catabolism of glucose via glycolysis and the tricarboxylic acid (TCA) cycle (Fig. 4; Supplementary Data 11). In addition, all three species have all the enzymes necessary to convert fructose and mannose to glucose, and to synthesize and utilize glycogen and trehalose. However, key enzymes that convert galactose and sucrose to glucose are missing. Additional notable losses include two enzymes for glyoxylation ${ }^{8}$, one key enzyme for gluconeogenesis, and all enzymes for pyruvate fermentation. These findings, together with the identification of almost all genes involved in oxidative phosphorylation (Supplementary Data 16), suggest that energy production in Pneumocystis largely relies on glucose through oxidative pathways.

Lipid metabolism genes in Pneumocystis are also greatly reduced in number, thus resulting in distinctive differences in predicted lipid content compared with other fungi (Fig. 4; Supplementary Data 12). All three Pneumocystis species are able to synthesize fecosterol and episterol, but unable to convert them to ergosterol, which potentially accounts for the resistance of Pneumocystis to classical antifungal agents as noted previously ${ }^{11}$. Moreover, only $P$. jirovecii but not $P$. murina or $P$. carinii can synthesize cholesterol ${ }^{13}$ (Supplementary Fig. 11). These findings support the hypothesis that $P$. murina and $P$. carinii, but not necessarily $P$. jirovecii, may scavenge cholesterol from their hosts $^{31}$, though the genes involved in sterol uptake have not been identified. Utilization of cholesterol rather than ergosterol may be contributing to a less rigid cell wall, thus allowing development of the trophic form of Pneumocystis since cholesterol-containing membranes are more flexible than ergosterol-containing

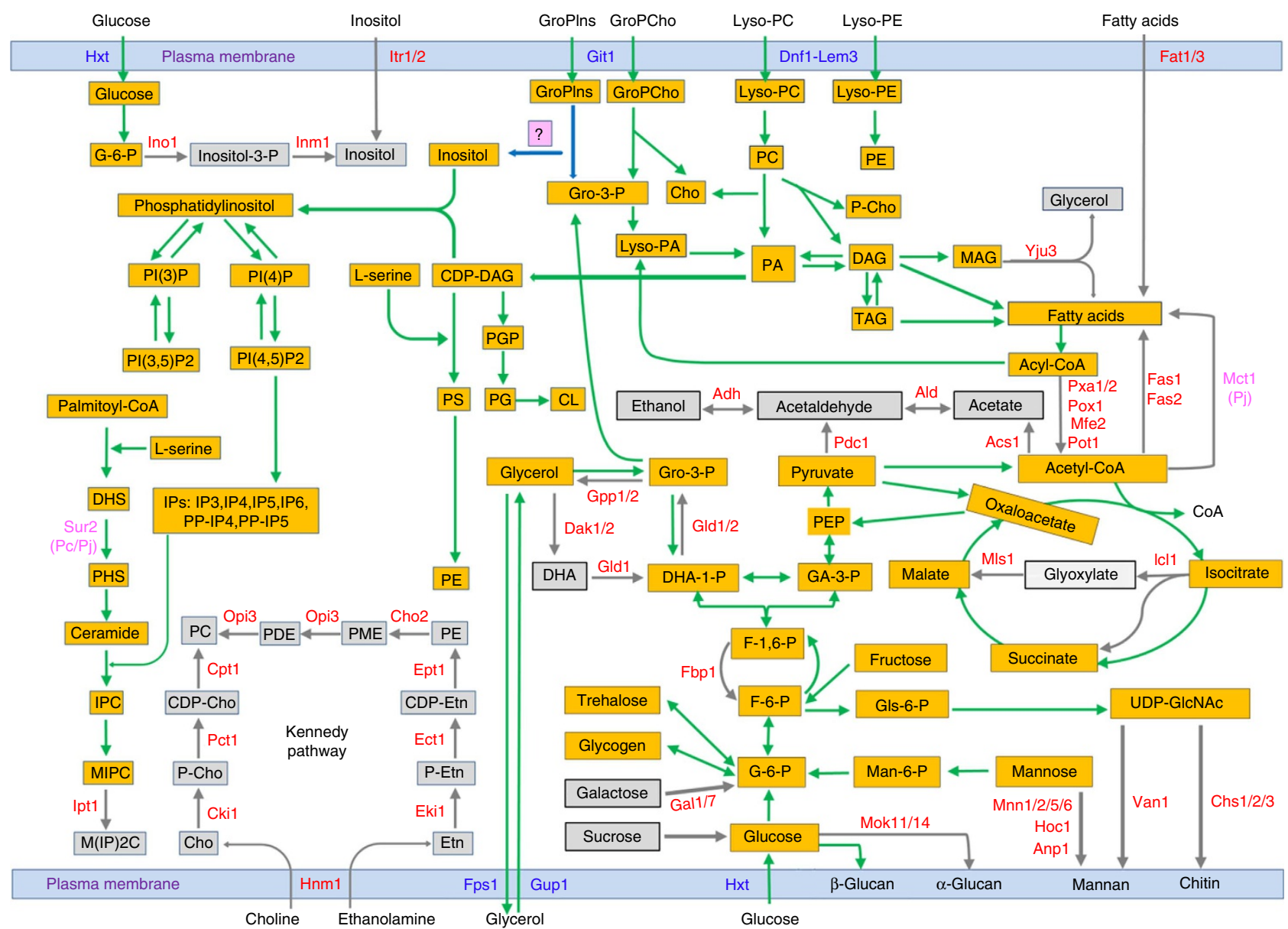

Figure 4 | Reduction of carbohydrate and lipid metabolism in Pneumocystis. A condensed version of pathways highlights retained (green arrows) and lost (grey arrows) pathways. Enzymes and membrane transporters absent in all three Pneumocystis species are highlighted in red font; those retained in all three species are highlighted in blue. Yellow and grey boxes indicate metabolites present and absent, respectively. Some metabolites are included more than once as they interact with multiple pathways, in which case the yellow or grey colouring refers to their role in different pathways. Enzyme Sur2 (in pink) is present in only $P$. murina but not $P$. carinii or $P$. jirovecii. Enzyme Mct1 (in pink) is present in both $P$. murina and $P$. carinii but not $P$. jirovecii. The boxed question mark ('?') leading to inositol indicates a hypothetical enzyme. The names of enzymes, transporters and metabolites follow the standard abbreviated names for S. cerevisiae. The enzyme and transporter names containing two or more digits represent duplicated enzymes and transporters. 


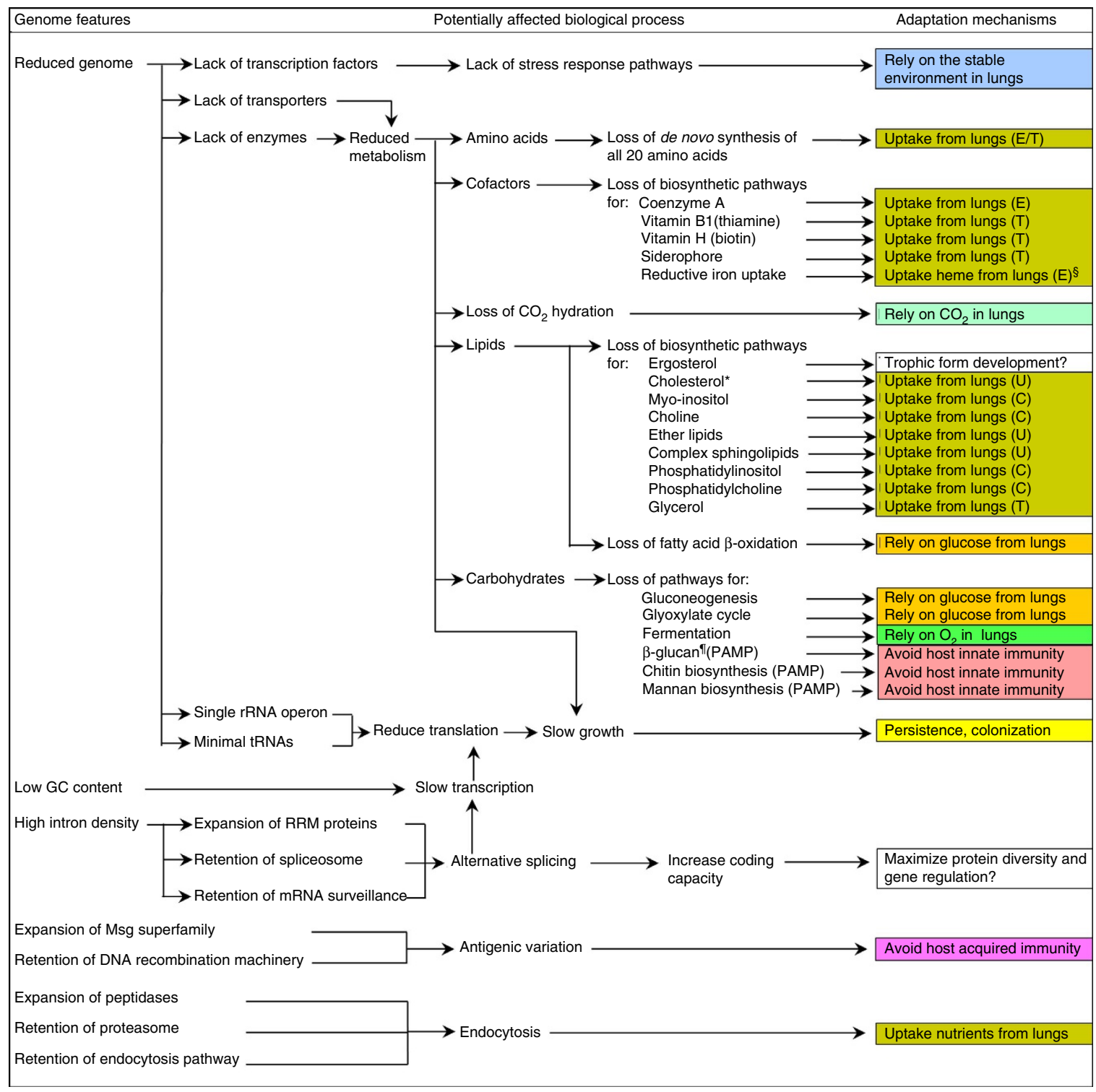

Figure 5 | Summary of mechanisms of adaptation to host lungs by Pneumocystis. Different mechanisms are highlighted by different colours. Potential mechanisms of uptake of nutrients (which cannot be synthesized de novo) include the use of plasma membrane-localized transporters (indicated by $\mathrm{T}$ ), conversion of other metabolites scavenged from hosts (indicated by $\mathrm{C}$ ), endocytosis (indicated by E) and unknown (indicated by $\mathrm{U}$ ). \$Potential uptake of haem or haemoglobin from lungs by endocytosis mediated by CFEM domain-containing proteins. ${ }^{*}$ Cholesterol biosynthesis pathway is retained in $P$. jirovecii but lost in P. murina and P. carinii. " $\beta$-glucan is present in cysts and absent in trophic forms. $\beta$-glucan as well as chitin and mannan in other fungal pathogens are known pathogen-associated molecular patterns (PAMP) involved in host immune recognition; none of these components is detected in Pneumocystis organisms except for the presence of $\beta$-glucan in the cyst form (Figs 6 and 7; Supplementary Fig. 13). Pneumocystis is the only fungus identified to date that cannot synthesize chitin.

membranes. All three Pneumocystis species lack not only the de novo synthesis pathways for myo-inositol, choline, complex sphingolipids, ether lipids, phosphatidylinositol, phosphatidylcholine and fatty acids (the cytosolic pathway involving fas 1 and fas 2 genes) but also the transporters for direct uptake of these lipids from external sources (Fig. 4; Supplementary Data 12). Nevertheless, alternative mechanisms could supply cells with phosphatidylinositol, phosphatidylcholine, inositol and choline (Fig. 4; Supplementary Note 5).

Strikingly, most of the genes involved in fatty acid $\beta$-oxidation are missing in all three species, suggesting that fatty acids are not an energy source for Pneumocystis, further supporting a high reliance on glucose as the main energy source. Of note, all three Pneumocystis species lack the enzymes for synthesis of glycerol from glycerone-phosphate or monoacylglycerol but encode homologues of yeast proteins (Gup1 and Fps1) responsible for glycerol uptake and export (Fig. 4; Supplementary Fig. 9b). Although glycerol is the only non-sugar carbon source that can enter into the TCA cycle, and is also required for synthesis of glycerolphospholipids, these two transporters may also play an important role in maintaining osmotic balance given the loss of critical cell wall components as discussed below.

Cofactor metabolism is also largely reduced in Pneumocystis (Supplementary Data 13). All three Pneumocystis species are missing almost all enzymes required for de novo synthesis and membrane transport of pantothenate, but retain all enzymes needed to convert pantothenate to $\mathrm{CoA}$, as well as a mitochondrial carrier protein (Leu5) for CoA (Supplementary Fig. 10), implying possible scavenging of pantothenate or its downstream metabolites from the host by other mechanisms (for example, 
endocytosis. Fig. 5; Supplementary Data 14). All three Pneumocystis species lack enzymes required for de novo synthesis of vitamins $\mathrm{B} 1$ and $\mathrm{H}$, as well as ubiquinone and siderophores, but have a potential plasma membrane transporter for each of these cofactors (Supplementary Data 13; Fig. 4; Supplementary Fig. 9c,d). P. jirovecii also lacks enzymes for de novo synthesis of $\mathrm{NAD}$, while retaining one salvage pathway using exogenous nicotinic acid mononucleotide imported by a transporter (Tna1). In contrast, both $P$. murina and $P$. carinii have all the enzymes and the transporter needed for de novo synthesis and salvage of NAD. All three Pneumocystis species show a near complete absence of proteins necessary for reductive iron assimilation and siderophore biosynthesis ${ }^{32}$ (Supplementary Data 13). Since each Pneumocystis species encodes five proteins with cysteine-rich CFEM domains (Supplementary Fig. 8), it is possible that, like Candida albicans ${ }^{33}$, Pneumocystis is able to scavenge iron from host haem and haemoglobin using one or more of these proteins as an extracellular haem receptor.

Our analysis suggests that endocytosis may serve as a mechanism for Pneumocystis to obtain nutrients in the absence of multiple different receptors. In support of this hypothesis, we found that each Pneumocystis species encodes nearly all proteins involved in clathrin-dependent endocytosis (Supplementary Data 14). In addition, genes encoding various degradative enzymes (including proteases, lipases, ATPase families and proteasome shown in Supplementary Data 3,5,12 and 15) and transporters localized in mitochondria and vacuoles are expanded or retained, and some of them are highly expressed (Supplementary Data 20), while biosynthetic pathways for many nutrients (including amino acids, lipids and cofactors, as noted above) and plasma-membrane-associated transporter families are completely lost or reduced. Loss of these transporters may reflect low concentrations of their targets in the host lung milieu.

Lack of chitin, outer chain $\mathrm{N}$-mannans and $\alpha$-glucan in cell wall. Fungal cell walls typically are composed primarily of chitin, chitosan, glucans, mannans and glycoproteins, which are covalently cross-linked together to protect the cell from changes in environmental stresses, while allowing the organism to interact with its environment. The structure and biosynthesis of such cell walls are unique to fungi and thus serve as an excellent target for antifungal agents. The formation and assembly of the Pneumocystis cell wall remain poorly understood, though numerous studies have found it to be rich in glycoproteins and, in cysts only, $\beta$-glucans ${ }^{34,35}$.

Remarkably, none of the three Pneumocystis species encodes the key enzyme chitin synthase required for chitin synthesis, or chitinases involved in chitin degradation during cell wall remodelling (Fig. 6; Supplementary Data 17). The absence of these two gene families strongly suggests that Pneumocystis does not contain chitin in its cell wall. While each Pneumocystis genome does encode homologues of a few accessory proteins not directly involved in chitin synthesis or degradation (such as Chs5 reported elsewhere ${ }^{36}$ ), none of these shares any signature protein domains found in chitin synthase or chitinase (Supplementary Fig. 12). In Saccharomyces cerevisiae, Chs5 functions as a component of the exomer complex involved in export of chitin synthase and other membrane proteins ${ }^{37}$; in Pneumocystis Chs5 could serve only the latter function, given the absence of chitin synthase. While the detection of chitin in P. carinii has been previously reported ${ }^{36,38}$ based on reactivity with non-specific lectins such as wheat germ agglutinin, this staining may be detecting other molecules based on the absence of chitin synthases in the genome.

We assayed for the presence of chitin in the Pneumocystis cell wall using a recombinant chitin-binding domain (CBD). While we found strong reactivity with Candida cell walls by in situ labelling, reactivity with Pneumocystis was totally absent (Fig. 6). In addition, we directly examined the cell wall content of partially purified Pneumocystis organisms and cultured S. cerevisiae cells using mass spectrometric analysis. No chitin-related oligosaccharides were identified in Pneumocystis, while they were detected in $S$. cerevisiae; glucan-related oligosaccharides were detected in both (Supplementary Note 6). The combination of these genomic and experimental results demonstrates that Pneumocystis is the first identified member of the fungal kingdom that does not have chitin.

$\beta$-glucans have been identified in the wall of the cyst form of Pneumocystis, but are absent from the more abundant trophic form $^{34,39}$. Pneumocystis genomes encode all the enzymes required for $\beta-1,3$ - and $\beta$-1,6-glucan synthesis and degradation ${ }^{34,39-41}$ (Supplementary Data 18). However, Pneumocystis species do not have any genes involved in synthesis and degradation of $\alpha$-glucan, which has been found in many fungi, and which can block innate immune recognition by the $\beta$-glucan receptor ${ }^{42}$.

Although all Pneumocystis species have abundant surface glycoproteins, especially Msg, little is known about their glycosylation state and other post-translational modifications. We found that Pneumocystis species encode enzymes required for synthesis of the $\mathrm{N}$ - and O-linked glycan core structure (containing up to nine mannose residues), which are localized to the endoplasmic reticulum, but lack genes for enzymes residing in the Golgi apparatus, which add mannose outer chains, including all the enzymes comprising mannan polymerase complex I and complex II, and $\alpha-1,6-, \quad \alpha-1,2-$ and $\alpha$-1,3-mannosyltransferase (Supplementary Data 19). The absence of these enzyme genes suggests that, unlike other fungi, Pneumocystis cell wall proteins including Msg are not highly mannosylated. N-linked profiling of PNGase-F-released $\mathrm{N}$-glycans showed that $\mathrm{M} 5 \mathrm{~N} 2$ is the predominant $\mathrm{N}$-linked glycan on $P$. carinii Msg proteins (Supplementary Fig. 13; Supplementary Note 7). Although trace amounts of M6N2 to M9N2 were detected as minor components, mannan type $\mathrm{N}$-glycans with more than nine mannose residues were not detected. Moreover, glycopeptide mapping of Msg tryptic digest by using liquid chromatography-tandem mass spectrometry (LC-MS/MS) identified $31 \mathrm{~N}$-linked glycans in $15 \mathrm{Msg}$ isoforms, all of which carried M5N2 as the predominant component and only 1 of which carried M6N2 as an additional, minor component (Fig. 7; Supplementary Fig. 13; Supplementary Data 21). The lack of $\mathrm{N}$-linked outer chain mannan may allow the organism to avoid recognition by innate immune responses, since in Candida such mannosylation is required for recognition by dectin-2, DC-SIGN and the macrophage mannose receptor of dendritic cells, while mutants that can synthesize only the core structure are poorly recognized $^{43,44}$

Transcription enrichment during infection. The expression level of each annotated gene was estimated using RNA-Seq data from three heavily infected animals each for $P$. murina and $P$. carinii. Overall, we find evidence of expression for nearly all genes (99\%, see Methods) but variation in expression level over 5 orders of magnitude. Using Gene Set Enrichment Analysis (GSEA ${ }^{45}$ ), we identified functional categories that were enriched in highly expressed genes in each species (Supplementary Data 20; Supplementary Fig. 14). The most highly enriched categories include Msgs and predicted secreted proteins (24-25 other than Msgs in each species). The enrichment of the latter suggests that additional secreted proteins may play an important role during infection. In addition, many functions involved in general metabolism of RNA and proteins, including the RNA RRM and 
a

\begin{tabular}{llll}
\hline & $\begin{array}{l}\text { Chitin } \\
\text { synthase }\end{array}$ & Chitinase & $\begin{array}{l}\text { Accessory } \\
\text { proteins }\end{array}$ \\
\hline P. murina & No & No & Yes \\
P. carinii & No & No & Yes \\
P. jirovecii & No & No & Yes \\
\hline T. deformans & I, III & Yes & Yes \\
S. pombe & I, II & Yes & Yes \\
S. octosporus & I, II & Yes & Yes \\
S. cryophilus & I, II & Yes & Yes \\
S. japonicus & I, II & Yes & Yes \\
\hline S. cerevisiae & I, II, IV & Yes & Yes \\
C. albicans & I, II, IV & Yes & Yes \\
\hline E. cuniculi & IV & No & No \\
E. intestinalis & IV & No & No \\
\hline
\end{tabular}

b
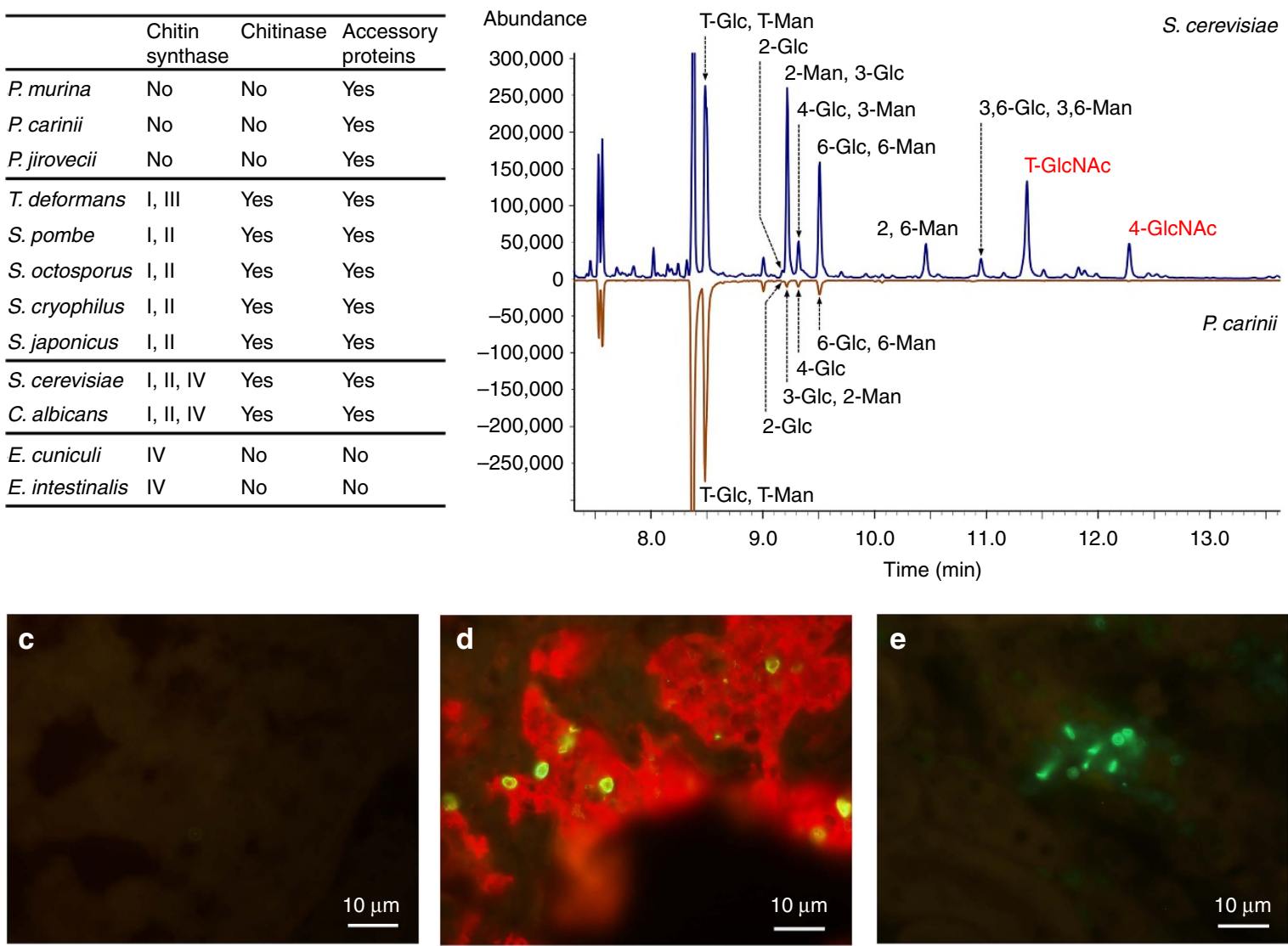

Figure 6 | Analysis of chitin in Pneumocystis and related fungi. (a) Enzymes and accessory proteins involved in chitin metabolism in fungi. Pneumocystis genomes do not encode any chitin synthase or chitinase, which are present in other fungi, but retain genes encoding four accessory proteins (Supplementary Data 17). (b) Gas chromatograms of partially methylated alditol acetates of $P$. carinii and S. cerevisiae (control) cell walls. Terminal and 4-linked N-acetylglucosamine signals (T-GIcNAc and 4-GIcNAc in red font) were detected in S. cerevisiae but not in P. carinii. Glucose (GIc) and mannose (Man) signals were detected in both species. (c-e) Detection of chitin with recombinant chitin-binding domain (Alexafluor 488) using P. murina-infected lung tissue (c) and C. albicans-infected kidneys (e) as a positive control. Pneumocystis organisms are demonstrated in $\mathbf{d}$ by dual staining with anti-Msg (red), which labels both trophic forms and cysts, and a dectin-Fc construct (green), which labels $\beta-1,3-$ glucan in cysts. Chitin staining is absent in $P$. murina but readily detected in $C$. albicans, while $\beta-1,3$-glucan is easily seen in $P$. murina. Original magnification, $\times 400$; scale bar, $10 \mu \mathrm{m}$.

LSM domain, are enriched among highly expressed genes in Pneumocystis.

Each Pneumocystis genome contains an exceptionally high intron density, with an average of 5 introns per gene, similar to only a few fungal genomes such as Cryptococcus with high levels of splicing and only slightly fewer than that in mammalian genomes (7-9 introns per gene) ${ }^{46}$. This is unusual for highly compacted fungal genomes where intron loss is usually observed, as in $S$. cerevisiae ${ }^{47}$ and Microsporidia ${ }^{48}$. The transcription and splicing process for genes with many introns requires higher energy and cellular resources; this appears correlated to the relative expansion and high-level expression of RRM domaincontaining genes (Fig. 2; Supplementary Fig. 14; Supplementary Data 6) and genes involved in spliceosome and mRNA surveillance in all Pneumocystis genomes (Supplementary Data 22). Using the RNA-Seq data, we identified high-level alternative splicing events in $P$. murina and $P$. carinii, with intron retention being the most common (detected for $42-49 \%$ of introns) and other types being infrequent ( $\leq 3 \%$ of introns for each type) (Supplementary Data 23). We assembled full-length alternatively spliced isoforms without premature termination codons for 263 and 275 genes of $P$. murina and $P$. carinii, respectively, though there is no functional enrichment of these genes. The high rate of intron retention correlates with the presence of all the components of the nonsense-mediated mRNA decay machinery in each Pneumocystis species (Supplementary Data 22). Alternative splicing of intron-containing genes could increase transcript diversity and regulate gene transcription or mRNA stability in this otherwise reduced genome, as suggested in earlier studies of $P$. carinii ${ }^{49}$ and other organisms ${ }^{50}$.

Adaption to a host lung environment. Our genome analysis has revealed new insights into the dependence of Pneumocystis on mammalian hosts. Pneumocystis has been identified almost exclusively in the lungs of humans and other mammals, where it remains extracellular but preferentially attaches to type I pneumocytes. Although an environmental reservoir has been hypothesized, there is no convincing evidence of such a reservoir; the current genome data strongly suggest that the entire life cycle occurs in the host. Genes involved in mating and meiosis are present and transcribed in all three Pneumocystis species (Supplementary Data 24), suggesting that sexual reproduction is actively occurring in lung tissue, as postulated in previous studies 9,11 . Thus, Pneumocystis presumably must obtain nutrients and proliferate in the lung environment, and at the same time it must withstand host defenses for sufficient periods to allow direct transmission to another susceptible host. 
a

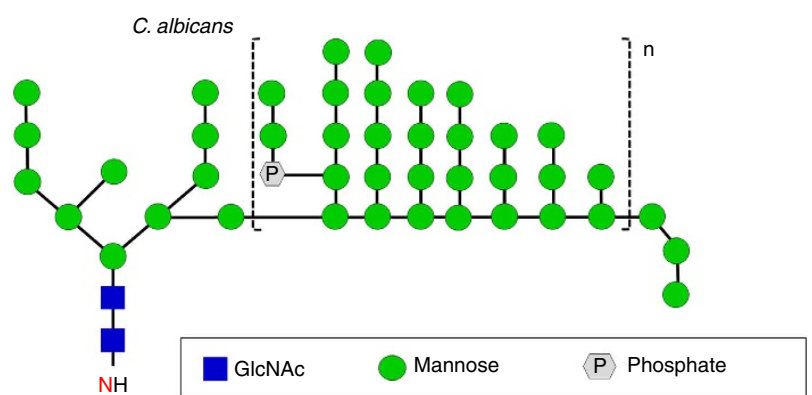

c

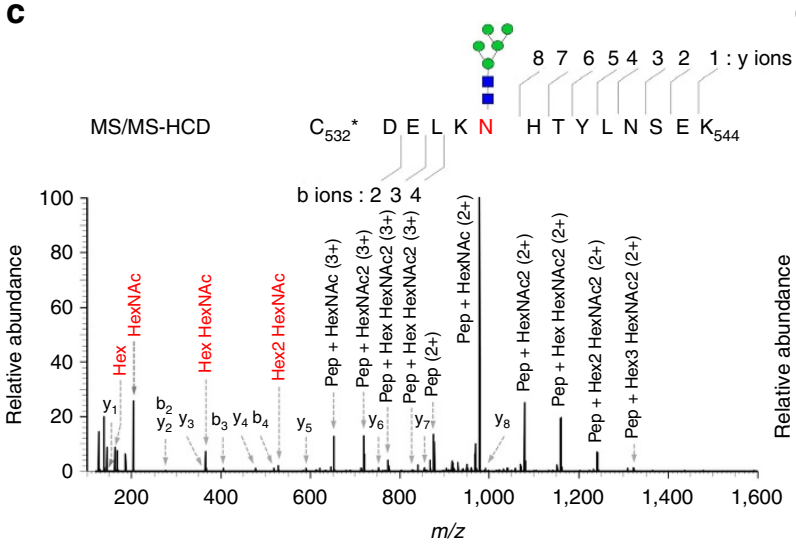

\section{d}

b

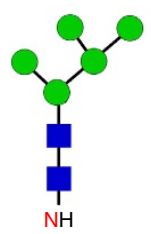

M5N2
Pneumocystis

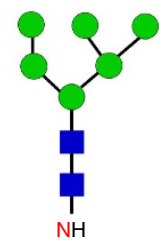

M6N2

MS/MS-ETD

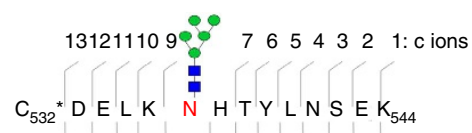

zions: $12345 \quad 678910111213$

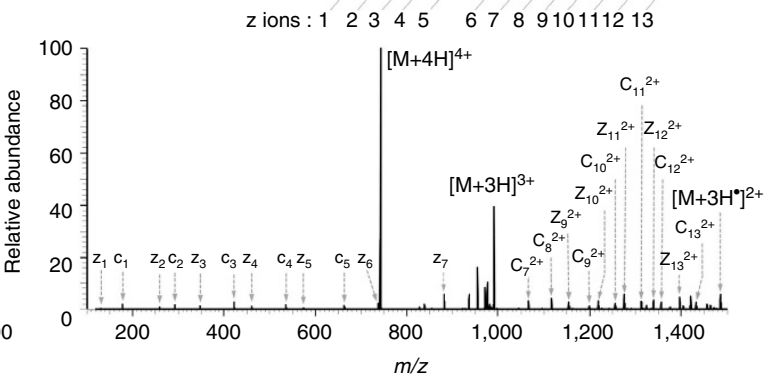

Figure 7 | Lack of hyper-mannose (mannan) glycosylation in Pneumocystis. (a) Diagram of N-linked mannan structure in C. albicans, based on ref. 43. (b) Diagram of $\mathrm{N}$-linked glycans in Pneumocystis, which lack the $\alpha$-1,6-linked mannose backbone as well as $\alpha-1,2-$ and $\alpha-1,3-$ linked mannose outer chains seen in C. albicans (square brackets). (c,d) Representative results of tandem mass spectrometry (MS/MS)-higher energy collisional dissociation (HCD) and electron transfer dissociation (ETD) analysis of an N-linked glycopeptide carrying Hexose5 HexNAc2 (M5N2) from one Msg isoform (T552_03736) in P. carinii. (c) MS/MS-HCD spectrum of glycopeptides showing the detection of glycan oxonium ions in the low mass region at $\mathrm{m} / \mathrm{z} 163.0603,204.0868$, 366.1398 and 528.1929 (indicated in red font). A series of fragment ions dues to neutral loss of the glycan moiety were observed as the main fragment ions in the HCD spectrum. Trace amounts of y-type and b-type peptide fragment ions were detected, confirming the sequence of the peptide backbone. (d) MS/MS-ETD spectra of peptide fragment ions with minimal neutral loss of glycan moiety. All expected peptide c-type and z-type fragment ions were detected except $c 8$ fragment ion, confirming the peptide sequence with high confidence, as well as the site and mass of the glycosylation modification.

During the process of adaption as an obligate pathogen, the Pneumocystis genome has contracted substantially compared with other fungi, though not to the extent of Microsporidia, which are intracellular organisms, while Pneumocystis are exclusively extracellular. The retention of all components for glucose uptake and catabolism while the glyoxylate and gluconeogenesis pathways were lost strongly suggests a high dependency on glucose from the host as the primary energy and carbon sources. Transcriptional regulation is also greatly simplified, implying that the host lung provides an optimal environment for Pneumocystis, with little need for sensing and responding to stress, as evidenced by the loss of genes involved in the $\mathrm{pH}$ sensing, osmotic and oxidative stress responses and cAMP-mediated signalling (Supplementary Data 25). The loss of the pyruvate fermentation pathway and other genes known to be essential for anaerobic growth (Supplementary Data 11 and 26) likely reflects adaptation exclusively to aerobic respiration in the lung environment with a stable oxygen supply. Another very unusual loss in Pneumocystis is the gene encoding carbonic anhydrase (Supplementary Data 9), which catalyses the interconversion between $\mathrm{CO}_{2}$ and bicarbonate, a key mechanism for providing bicarbonate to cells and for regulation of intracellular $\mathrm{pH}$, and which is widely distributed in all life kingdoms ${ }^{51}$. This enzyme may be unnecessary in the $\mathrm{CO}_{2}$-rich lung environment (5-6\%), while its absence suggests Pneumocystis is unable to survive under ambient air conditions $\left(0.03-0.04 \% \mathrm{CO}_{2}\right)$.

\section{Discussion}

Our study suggests that Pneumocystis is highly adapted to existence in the host lung, with strict dependence on the mammalian host for nutrients and a stable environment, while utilizing efficient strategies for immune evasion, thus facilitating colonization and persistence in its host. Among the most striking findings in this study is the loss of two important fungal cell wall components, chitin and outer chain N-mannans, both predicted by genome analysis and further supported by biochemical assays. Furthermore, $\beta$-1,3-glucan has previously been shown to be absent from the cell wall of the trophic form of Pneumocysti ${ }^{34,39}$, which is $\sim 10$ - to 20 -fold more abundant than the cyst form. While $\beta$-glucan is present and exposed in some Pneumocystis cysts, we have found that in most cysts the $\beta$-1,3-glucan is masked by Msg and other surface proteins (unpublished observations). Given that chitin, $\beta$-glucans and mannan are all known pathogen-associated molecular patterns (PAMPs) that trigger innate host defense responses through interactions with host pattern recognition receptors (PRRs) such as dectin-1 and DC-SIGN $^{43,52}$, their simultaneous absence or masking may be beneficial in evading or delaying recognition of Pneumocystis, especially the trophic form, by the innate immune system. Antigenic variation provided by Msg isoforms extends potential immune evasion to include adaptive immune responses. Retention of $\beta$-glucan in cysts presumably is critical to organism survival, possibly by providing a rigid spherical cell 
wall that is aerodynamically efficient for transmitting infection to other hosts, while protecting the organism from the harsher environmental conditions outside the lung ${ }^{5}$. The absence of chitin and $\beta$-glucan on the cell wall (Fig. 6) suggests that trophic forms are more fragile than other fungi. Greater malleability of the trophic cell wall could facilitate intimate contact with host cell membranes, and improve the ability of Pneumocystis to obtain nutrients by direct cell-to-cell contact or by endocytosis. The evolutionary adaptation by Pneumocystis is in sharp contrast to other pathogenic fungal species that normally live outside the mammalian host while being able to infect different host species and different tissues.

The availability of three high-quality Pneumocystis genomes provides important information about the biology of these species, and potentially provides insights that will guide developing in vitro culturing of this organism, a critical goal of the Pneumocystis research community. Moreover, given the loss of many biosynthetic and metabolic pathways, the remaining pathways, including the limited number of transporters (Supplementary Table 4), provide attractive targets for anti-Pneumocystis drug development.

\section{Methods}

Pneumocystis organisms from rodents and humans. P. murina-infected lung samples were obtained from CD40 ligand knock-out female mice ${ }^{26}$. P. cariniiinfected lung samples were obtained from immunosuppressed Sprague-Dawley male rats $^{24}$. P. jirovecii-infected autopsy lung samples were from one patient with AIDS, who was infected with only one $P$. jirovecii strain based on genotyping at five different genomic loci ${ }^{53}$. Animal and human subject experimentation guidelines of the National Institutes of Health were followed in the conduct of these studies.

Preparation of genomic DNA samples. Pneumocystis-infected lung tissues were cut into small pieces, homogenized in Qiagen Tissuelyser ( 5 times for $20 \mathrm{~s}$ at 1/30 frequency), then centrifuged at $15,000 \mathrm{~g}$ for $6 \mathrm{~min}$. The pellet was resuspended in Trypsin/EDTA Solution (Lonza), incubated at $37^{\circ} \mathrm{C}$ for $30 \mathrm{~min}$ and centrifuged at $15,000 \mathrm{~g}$ for $6 \mathrm{~min}$. The pellet was washed once in PBS, resuspended in lysis buffer containing $2.9 \%(\mathrm{w} / \mathrm{v})$ collagenase type I (Gibco) and $0.1 \%(\mathrm{w} / \mathrm{v})$ DNase I (Sigma), incubated at $37^{\circ} \mathrm{C}$ for $30 \mathrm{~min}$, and centrifuged at $15,000 \mathrm{~g}$ for $6 \mathrm{~min}$. This sequential enzyme digestion was expected to remove most of the host cells and DNA as well as potentially some of Pneumocystis trophic forms. The pellet was washed three times in PBS, resuspended in $100 \mu \mathrm{l}$ of $0.5 \%$ (w/v) Zymolyase solution (Zymo Research) and incubated at $37^{\circ} \mathrm{C}$ for $1 \mathrm{~h}$. Genomic DNA was extracted using the MasterPure Yeast DNA Purification Kit (Epicentre). RNA was removed using DNase-free RNAase (Epicentre).

All DNA samples were analysed by quantitative real-time PCR (qPCR) assays using fluorescence resonance energy transfer probes ${ }^{54}$ to measure the fraction of Pneumocystis DNA compared with host DNA in each sample. In qPCR for both P. murina ${ }^{20}$ and $P$. carinii ${ }^{54}$, the target was the single-copy dihydrofolate reductase (dhfr) gene of Pneumocystis and the target for the host was a highly conserved region of the single-copy polycystic kidney disease 1 ( $p k d 1)$ gene $^{22}$. The targets for $P$. jirovecii and human qPCR were the $m s g$ gene family ${ }^{55}$ and the single-copy $\beta$-globin gene ${ }^{56}$, respectively. The purity of each DNA sample was estimated by the ratio of Pneumocystis to host genome copy number and based on an estimated genome size of $8 \mathrm{Mb}$ for Pneumocystis and 2.7-3.3 Gb for the host. The qPCR results were validated by 454 or Illumina MiSeq sequencing of selected samples. DNA samples extracted from enriched $P$. murina or $P$. carinii preparations contained up to $90 \%$ Pneumocystis DNA, and from enriched $P$. jirovecii preparations, $10-25 \% P$. jirovecii DNA, while those extracted directly from heavily infected lung tissues contained $<0.4-1 \%$ Pneumocystis DNA.

P. murina genome sequencing and assembly. To assess the quality of the $P$. murina DNA preparations for whole-genome sequencing, small-scale sequencing was first conducted using a 454 GS FLX Titanium Sequencer (Roche Applied Science) at Leidos, Inc. (Frederick, MD) according to the 454 standard shotgun sequencing protocols. A total of 4 million reads (with a mean length of 344 bases) were generated, with $\sim 40 \%$ of them having blast hits with $P$. carinii ${ }^{7}$. Subsequently, deep sequencing was performed using Illumina HiSeq technology at the Broad Institute (Cambridge, MA). Seven different P. murina DNA preparations (each with $60-90 \%$ purity for $P$. murina by qPCR) were used to construct small insert libraries; each was sequenced, and the library (preparation B123 from a single mouse, center project G11228) with the lowest percent of contaminating host mouse DNA was used for assembly. From this library, a total of 34 million 101 base paired-end reads with a mean insert size of 153 bases were generated. A second larger insert library (preparation C2 from another mouse, center project
G11230) with a mean insert size of 1,247 bases was prepared, and a total of 83 million 101 base paired-end reads were generated. After removal of mouse sequences (version $\mathrm{mm} 9$ ) and $P$. murina mitochondrial sequences ${ }^{22}$, these reads were assembled with Allpaths (version R37380) with default parameters ${ }^{57}$; the resulting assembly was screened for contaminating sequences (mouse and bacteria) and to remove $P$. murina mitochondrial sequences ${ }^{22}$ by aligning to the nonredundant nucleotide database from NCBI and by removing the scaffolds with high coverage that matched non-fungal organisms. The draft assembly of 24 scaffolds was further improved using long 454 reads and PCR to support scaffold joins. The final assembly contained 17 scaffolds. All internal contig gaps were closed by PCR and Sanger sequencing. The ends of scaffolds without $m s g$ genes or telomere repeats were extended by PCR and/or PacBio sequencing (Supplementary Methods).

P. carinii genome sequencing and assembly. After preliminary 454 sequencing demonstrated a purity of 63\%, 1 P. carinii DNA preparation (no. B80 from 2 heavily infected rats, $80 \%$ purity for $P$. carinii by qPCR) was used to construct 2 libraries with a mean insert size of 180 bases and 793 bases, respectively. Each was sequenced using 101 base paired-end reads on the Illumina MiSeq platform at the Broad Institute, and, after removal of host rat sequences and P. carinii mitochondrial sequences ${ }^{22}$, assembled using AllPaths-LG (version R47825) with default parameters, resulting in a total of 53 scaffolds. After joining scaffolds using long 454 reads and confirming by PCR, the final genome assembly contained 17 scaffolds. All internal contig gaps were closed by PCR and Sanger sequencing. The ends of six scaffolds overlapped with the seven telomere sequences reported elsewhere ${ }^{58}$; they were merged and confirmed by PCR and/or aligning merged scaffolds with Illumina and 454 raw reads. The ends of several scaffolds without $m s g$ genes, kexin genes or telomere repeats were extended by PCR and/or PacBio sequencing (Supplementary Methods).

P. jirovecii genome sequencing and assembly. Three enriched DNA samples (RU7, RU12 and RU817) from a single autopsy lung sample RU (1.4-2 $\mu$ g each, with $10-25 \%$ P. jirovecii DNA by qPCR) were used to construct three libraries (156 base average insert size); each was sequenced on the Illumina HiSeq platform at the Broad Institute. To improve on initial assemblies with high numbers of contigs, additional sequence was generated from hybrid-selected DNA samples as previously described ${ }^{59} ; 120$ base oligo bait probes were designed to target regions present in the genome assembly as well as to pull in uncovered regions and transcripts; probes were designed for: existing assemblies ${ }^{8}$ including higher probe density at contig ends, unassembled reads $^{8}$ that did not match the human genome assembled RNA-Seq transcripts ${ }^{8}$ that did not match existing assemblies or the human genome and unanchored $m s g$ sequences ${ }^{25}$. Host sequences were removed by aligning to human genome assembly 19 (GRCh37/hg19), and $P$. jirovecii mitochondrial sequences ${ }^{22}$ were removed. The remaining 101 base paired-end reads were assembled separately with Spades 2.5.1 (at the Broad Institute), Spades 3.0 (at Leidos) and Abyss (Biowulf at NIH), which resulted in 400, 149 and 312 contigs, respectively. All these contigs were merged with Sequencher (Gene Codes Co., Ann Arbour, MI), resulting in a total of 20 scaffolds. All internal gaps were closed by PCR and Sanger sequencing. Scaffolds were validated by examining raw reads aligned by Seqman Pro (DNAStar, Madison, WI) to ensure there were no false joins. To improve the coverage of $m s g$ genes in the assembly, PacBio sequencing was performed as described in Supplementary Methods.

Chromosome electrophoresis and Southern hybridization. To compare the $P$. murina assembly with chromosome number and length, we used contour clamped homogeneous electrical field (CHEF) electrophoresis to separate chromosomes. P. murina organisms were partially purified from fresh mouse lungs by Ficoll-Hypaque density gradient centrifugation ${ }^{60}$, and then processed for $\mathrm{CHEF}^{61}$ using the CHEF Yeast Genomic DNA Plug Kit (Bio-Rad). Briefly, partially purified $P$. murina organisms were embedded in $0.8 \%$ CleanCut agarose, treated with $24 \mathrm{U} \mathrm{ml}^{-1}$ proteinase $\mathrm{K}$ overnight at $50^{\circ} \mathrm{C}$, then washed four times in $1 \times$ Wash Buffer and stored at $4{ }^{\circ} \mathrm{C}$. Electrophoresis was performed in $1 \%$ agarose gels $(14 \times 21 \mathrm{~cm})$ using the CHEF DR II apparatus (Bio-Rad). Gels were run in $0.5 \times$ TBE buffer for $144 \mathrm{~h}$ at $135 \mathrm{~V}$ and $12.5^{\circ} \mathrm{C}$, with a $50 \mathrm{~s}$ initial pulse that was gradually increased to $100 \mathrm{~s}$. The gel was stained with ethidium bromide (Sigma), then DNA was transferred to Nytran membranes (Schleicher \& Schuell) under neutral conditions ${ }^{61}$. We used two blots prepared from the same DNA plug, and each blot was hybridized consecutively to different probes, with stripping of the blot between hybridizations. All probes were PCR-amplified double-stranded DNA fragments labelled using the PCR DIG Probe Synthesis Kit (Roche Applied Science) except for the probe for telomere repeats, which was a synthesized oligonucleotide labelled using the DIG Oligonucleotide Tailing kit (Roche Applied Science). All primer and probe sequences are provided in Supplementary Data 27. Hybridization and signal detection were performed by using the DIG Probe Hybridization system (Roche Applied Science) as described previously ${ }^{22}$.

RNA-Seq, expression levels and gene prediction. Three independent samples of $P$. murina and $P$. carinii organisms were partially purified from heavily infected lungs of three mice and rats, respectively, by Ficoll-Hypaque density gradient 
centrifugation ${ }^{60}$. The partially purified pellets were expected to contain both cyst and trophic forms. Total RNA was isolated using the RNeasy Mini Kit (Qiagen). The ratio of Pneumocystis to host RNA was estimated to be from 1:4 to 8:1 based on the density of RNA bands in agarose gels for the host 28S rRNA and Pneumocystis 26S rRNA. Strand-specific libraries were constructed using the dUTP second strand marking method ${ }^{62,63}$, except as noted below. For all samples, poly(A) RNA was purified using the Dynabeads mRNA Purification kit (Life Technologies), with two rounds of selection (with bead regeneration), resulting in $<5 \%$ rRNA as measured by the Bioanalyzer RNA 6000 Pico chip (Agilent) program. The resulting mRNA (200 ng per sample) was treated with the Turbo DNA-free kit (Ambion), checked by qPCR for the absence of detectable genomic DNA (Supplementary Table 5), and fragmented in $1 \times$ RNA fragmentation buffer (Affymetrix) for 4 min at $80^{\circ} \mathrm{C}$. After first-strand complementary DNA (cDNA) synthesis a $2.0 \times$ volume of RNAClean SPRI beads (Beckman Coulter Genomics) clean-up was used instead of phenol/chloroform/ isoamyl alcohol (25:24:1) extraction and ethanol precipitation. Size selection after adapter ligation was done with two $0.7 \times$ Ampure beads (Beckman Coulter Genomics) clean-up steps and eight cycles of PCR were used to generate the library. Libraries were sequenced on the HiSeq2000 platform generating an average of 26.1 million paired 76 base reads for each of the 6 samples.

To examine expression levels, RNA-Seq reads from each of the three samples for each organism were aligned to the extracted coding DNA sequences (CDSs) using bowtie ${ }^{65}$. The alignment bam files were then used to quantify transcript abundances by RSEM ${ }^{6}$. We selected the top $5 \%$ of expression levels to examine functional enrichment by GSEA ${ }^{45}$. For each organism, we examined GSEA of gene sets classified based on Pfam, TIGRFAM, KEGG and SignalP functional annotations. We also ran GSEA on some manually curated gene sets such as msg genes.

The relative expression level for each gene was expressed as fragments per $\mathrm{kb}$ of exon per million fragments mapped (FPKM). For both P. murina and P. carinii, $99 \%$ of all annotated genes were expressed (FPKM $>2$ ), with a median coverage of 151 FPKM in both species. Further, expressed genes showed good alignment depth across the transcript, with $98 \%$ of all genes in each species having at least fivefold RNA-Seq depth. All genes without detectable expression (26 in P. murina and 23 in $P$. carinii) contain short (average size of $\sim 250 \mathrm{bp}$, potentially pseudogenes) or incomplete CDSs.

The P. murina and P. carinii genomes were annotated using a combination of expression data (RNA-Seq), homology information and ab initio gene finding methods (Supplementary Methods) as previously described ${ }^{67}$. These methods were also used to annotate the RU assembly of $P$. jirovecii, with the exception that RNA-Seq data was not used (Supplementary Methods). Gene sets were compared based on functional annotation and used as the basis for syntenic and phylogenetic analysis (Supplementary Methods).

Analysis of strain variation in $\boldsymbol{P}$. jirovecii. To examine genome-wide single nucleotide polymorphisms (SNPs) between sequenced isolates, the RU7 assembly generated in this study was used as the reference compared with the SE8 raw reads $^{8}$. SE8 reads were retrieved from NCBI (ERR135854 to ERR135863) and converted to fastq format. Individual fastq files were concatenated, and the full read set aligned to the RU7 assembly using BWA-MEM ${ }^{68}$ and converted to sorted bam using SAMtools ${ }^{64}$. This resulted in very high alignment depth across all assembly bases, with a median alignment depth of 1,046 .

The Genome Analysis Toolkit ${ }^{69}$ (GATK) v2.7-4-g6f46d11 was used to call both variant and reference bases from the alignments. Briefly, the Picard tools (http://picard.sourceforge.net) AddOrReplaceReadGroups, MarkDuplicates, CreateSequenceDictionary and ReorderSamwere were used to preprocess the alignments, followed by GATK RealignerTargetCreator and IndelRealigner for resolving misaligned reads close to indels. Next, GATK UnifiedGenotyper (with the haploid genotype likelihood model (GLM)) was run with both SNP and INDEL GLM. We additionally ran BaseRecalibrator and PrintReads for base quality score recalibration on sites called using GLM SNP and re-called variants with UnifiedGenotyper emitting all sites. A final filtering step was used to remove any position that was called by both GLMs (that is, incompatible indels and SNPs) and to remove positions with low read depth support $(<10)$. The 24,902 SNPs were then mapped to the gene set predicted on the SE8 assembly; a total of 14,135 SNPs are located in CDS regions, with substitutions affecting protein coding regions as follows: 7,410 nonsynonymous, 6,690 synonymous, 26 nonsense and 9 extensions. SNP density across the SE8 assembly was calculated for $5-\mathrm{kb}$ windows.

Detection of chitin by fluorescence labelling. The cDNA sequence encoding the CBD of Bacillus circulans chitinase A1 gene ${ }^{70}(2,183-2,338$ bp, GenBank accession code M57601.1) was optimized for bacterial expression (GenScript USA Inc.) and modified by the addition of ATG and 3 HA tags. The modified sequence was synthesized (GenScript USA Inc., Supplementary Fig. 15) and cloned into pET-28 vector (EMD Biosciences). The cDNA construct was transformed into Escherichia coli strain BL21 (DE3) RIL (Agilent technologies) and expressed as a His tag fusion protein. The expressed protein was purified in two steps, first using anti-CBD antibody (New England Biolabs) that was immobilized using AminoLink Plus Immobilization Kit (Thermo Scientific), and then the Hispur cobalt purification kit (Thermo Scientific). The purified protein was biotinylated using Lightning-Link
Biotin conjugation kit (Type A) (Innova Biosciences Ltd) and used for fluorescence labelling (Histoserv, Inc., Germantown, MD) of P. murina-infected lung tissue sections fixed in Histochoice (Amresco, Inc.). Alexafluor 488-conjugated streptavidin was used for the detection of CBD. Pneumocystis organisms were detected by an anti-Msg antibody ${ }^{26}$ and cysts by a dectin-Fc recombinant protein (kindly provided by Dr Chad Steele, the University of Alabama at Birmingham, Alabama) $)^{71}$. Mouse kidney sections infected with Candida (kindly provided by Dr Michail Lionakis, the National Institute of Allergy and Infectious Diseases, Bethesda, Maryland) ${ }^{72}$ were used as a positive control for chitin staining (Fig. 5).

Chitin glycosyl linkage analysis. To prepare cell wall fraction, $P$. carinii organisms were partially purified from infected rat lungs by Ficoll-Hypaque density gradient centrifugation $^{60}$; S. cerevisiae organisms were obtained from Stratagene (strain YPH499) and grown in standard yeast culture. Cells were resuspended in denaturing buffer (2\% SDS, $1 \mathrm{mM}$ EDTA in $50 \mathrm{mM}$ Tris $\mathrm{pH} 8.0)$ and heated at $100^{\circ} \mathrm{C}$ for $10 \mathrm{~min}$. The cell wall pellet was recovered by centrifugation at $5,000 \mathrm{~g}$ for 5 min., washed twice with PBS, and dried in a speed vacuum concentrator.

For chitin analysis, cell wall pellets were incubated with $0.1 \%(\mathrm{w} / \mathrm{v})$ chitinase (Sigma) in $50 \mathrm{mM}$ sodium acetate buffer, $\mathrm{pH} 5.6$, at $37^{\circ} \mathrm{C}$ for $72 \mathrm{~h}$. Insoluble materials after the initial digestions were further treated sequentially with pronase (Roche), lyticase (Sigma) and chitinase to look for residual chitin. Protein digestion was performed using $0.01 \%(\mathrm{w} / \mathrm{v})$ pronase in Tris- $\mathrm{HCl}$ buffer $\mathrm{pH} 8.2$ with $10 \mathrm{mM}$ $\mathrm{CaCl}_{2}$ at $37^{\circ} \mathrm{C}$ for $48 \mathrm{~h}$. Glucan digestion was performed with $0.04 \%$ (w/v) lyticase in $50 \mathrm{mM}$ sodium phosphate buffer, $\mathrm{pH} 7.5$ at $37^{\circ} \mathrm{C}$ for $24 \mathrm{~h}$. Chitinase digestion was performed as above. After each digestion, the enzyme was inactivated by heating at $100^{\circ} \mathrm{C}$ for $5 \mathrm{~min}$.

Digested cell wall components were analysed by matrix-assisted laserdesorption ionization time-of-flight MS (MALDI/TOF-MS) and nanospray ionization MS (NSI-MSn). An aliquot of supernatant after each enzyme digestion was analysed by MS to determine oligosaccharide content. Prior to MS analysis, enzymes were removed by passing through a C18 Sep-PAK cartridge. The flow-through was collected in 5\% acetic acid and lyophilized. The dried samples were permethylated using previously published methods ${ }^{73}$ and profiled by MS. MALDI/TOF-MS was performed in the reflector positive ion mode using DHBA ( $\alpha$-dihyroxybenzoic acid, $20 \mathrm{mg} \mathrm{ml}^{-1}$ solution in $50 \%$ methanol/water) as a matrix. The spectrum was obtained by using an AB SCIEX TOF/TOF 5800 (AB SCIEX). NSI-MSn analysis was performed on an LTQ Orbitrap XL mass spectrometer (Thermo Fisher) equipped with a nanospray ion source. Permethylated sample was dissolved in $1 \mathrm{mM} \mathrm{NaOH}$ in $50 \%$ methanol and infused directly into the instrument at a constant flow rate of $0.5 \mu \mathrm{min}^{-1}$. A full Fourier transform MS spectrum was collected at 30,000 resolution. The capillary temperature was set at $210^{\circ} \mathrm{C}$ and MS analysis was performed in the positive ion mode. For total ion mapping (automated MS/MS analysis), an $\mathrm{m} / \mathrm{z}$ range of 200-2,000 was scanned with ion trap MS mode in successive 2.8 mass unit windows that overlapped the preceding window by $2 \mathrm{MU}$.

For determination of glycosyl linkages, partially methylated alditol acetates (PMAA) were prepared from fully permethylated glycans. The permethylated glycans were hydrolyzed with $\mathrm{HCl} /$ water/acetic acid $\left(0.5: 1.5: 8\right.$, by vol.) at $80^{\circ} \mathrm{C}$ for $18 \mathrm{~h}$, followed by reduction with $1 \% \mathrm{NaBD}_{4}$ in $30 \mathrm{mM} \mathrm{NaOH}$ overnight, then acetylation with acetic anhydride/pyridine $(1: 1, \mathrm{v} / \mathrm{v})$ at $100^{\circ} \mathrm{C}$ for $15 \mathrm{~min}$ and analysis by gas chromatograph-MS (GC-MS) on an Agilent 7890A GC interfaced to a 5975C MSD (mass selective detector, electron impact ionization mode). The separation of the PMAA was performed on a 30-m SP2331 fused silica capillary column (Supelco) for the neutral sugar derivatives, and a DB-1 (Agilent) column for amino sugar derivatives.

Msg protein glycosylation identification. To purify $P$. carinii Msg proteins, partially purified $P$. carinii organisms ${ }^{60}$ were resuspended in $2 \%$ SDS in $50 \mathrm{mM}$ Tris-HCl buffer, $\mathrm{pH} 8.0$, boiled for $10 \mathrm{~min}$ and centrifuged. The supernatant was collected. The extraction procedure was repeated two more times, the supernatants were pooled and SDS was removed using the SDS-Out SDS Precipitation Kit (Thermo Scientific). Solubilized Msg was affinity purified using an anti-Msg monoclonal antibody (RA-E7, gift of Drs Peter Walzer and Michael Linke ${ }^{74}$, the University of Cincinnati, Cincinnati, Ohio) immobilized on to AminoLink plus column (AminoLink plus immobilization kit) following manufacturer's instructions. The protein extract was diluted with an equal volume of Tris buffered saline and incubated with the immobilized antibody in the column by end-over-end mixing overnight at $4^{\circ} \mathrm{C}$. The column was then drained and washed with $0.1 \mathrm{M}$ sodium phosphate buffer $\mathrm{pH} 6.9$. The Msg was eluted with $150 \mathrm{mM}$ ammonium hydroxide and fractions were neutralized with $1 \mathrm{M} \mathrm{NaH} \mathrm{NO}_{4}$. The fractions containing Msg were pooled and concentrated using Microcon- $30 \mathrm{kDa}$ centrifugal filter unit (Millipore) and the buffer was exchanged to $0.1 \mathrm{M}$ sodium phosphate buffer $\mathrm{pH}$ 6.9. Aliquots were stored at $-80^{\circ} \mathrm{C}$.

Purified Msg proteins were subjected to glycopeptide detection by LC-MS. Glycoproteomic analysis was carried out as previously described ${ }^{75}$. Briefly, the tryptic digested peptides of purified Msg proteins were separated with a Magic C18 column $(15 \mathrm{~cm} \times 75 \mu \mathrm{m}$, Bruker-Michrom, CA) and analysed with an Orbitrap Fusion (Thermo Scientific) mass spectrometer after Msg was denatured, reduced, alkylated and desalted. Gradient elution was performed with a 30 min linear gradient of $5-35 \%$ acetonitrile in $0.1 \%$ formic acid at a flow rate of $300 \mathrm{nlmin}^{-1}$ 
The MS data were processed automatically by a data-dependent MS scan pipeline, which integrated three dissociation methods, including higher energy collisional dissociation (HCD), electron transfer dissociation (ETD) and collision-induced dissociation (CID). In this pipeline, the full MS spectrum was first acquired from most abundant ions between $\mathrm{m} / \mathrm{z} 350$ and $\mathrm{m} / \mathrm{z} 1,550$ with a 3 -second cycle time at 120,000 resolutions in FT mode. Subsequently the acquired MS data were assessed by MS/MS with HCD-product-dependent ETD or HCD-product-dependent ETD/CID mode with most abundant ions within 3-s cycle time. For the HCD-product ion trigger MS/MS, glycan oxonium ions at $\mathrm{m} / z 204.0867$ (HexNAc), $m / z 138.0545$ (HexNAc fragment) or $m / z 366.1396$ (Hexose-HexNAc) in the HCD spectra were used to trigger ETD or CID acquisition. The MS/MS were measured at a resolution of 15,000 .

For the mapping of glycopeptides from P. carinii Msg digests, LC-MS data were analysed using Byonic software (Protein Metrics) and the data annotation by the software was manually validated. Byonic parameters were set to allow 3.0 p.p.m. of precursor ion mass tolerance and 3.0 p.p.m. of fragment ion tolerance with monoisotopic mass. Digested peptides were allowed with up to three missed internal cleavage sites, and the differential modifications of 57.02146 and 15.9949 Da were allowed for alkylated cysteine and oxidation of methionine, respectively. Protein database used for protein blast included the protein set of the rat genome at NCBI (version 104) and of the P. carinii genome B80 generated in this study. Glycan database used was 309 mammalian N-glycan database (Default database in the Byonic software). Released N-linked glycan analysis of Msg was performed prior to the glycopeptide mapping.

Data annotation by the software was further filtered as follows. Any protein identification with FDR $>1 \%, \log$ probability $<4$ or best Byonic score $<500$ was excluded. Moreover, any glycopeptide spectra annotations with Byonic score $<400$ were excluded. Glycopeptide identifications that remained after these strict filters were manually validated by looking at fragment ions matched to the theoretical glycopeptide fragmentation, the presence of a series of expected glycan oxonium ions and neutral loss of the glycan moiety for MS/MS-HCD data.

\section{References}

1. Rabodonirina, M. et al. Molecular evidence of interhuman transmission of Pneumocystis pneumonia among renal transplant recipients hospitalized with HIV-infected patients. Emerg. Infect. Dis. 10, 1766-1773 (2004).

2. Sassi, M. et al. Outbreaks of Pneumocystis pneumonia in 2 renal transplant centers linked to a single strain of Pneumocystis: implications for transmission and virulence. Clin. Infect. Dis. 54, 1437-1444 (2012).

3. Morris, A. \& Norris, K. A. Colonization by Pneumocystis jirovecii and its role in disease. Clin. Microbiol. Rev. 25, 297-317 (2012).

4. Thomas, Jr C. F. \& Limper, A. H. Current insights into the biology and pathogenesis of Pneumocystis pneumonia. Nat. Rev. Microbiol. 5, 298-308 (2007).

5. Cushion, M. T. et al. Echinocandin treatment of Pneumocystis pneumonia in rodent models depletes cysts leaving trophic burdens that cannot transmit the infection. PLoS ONE 5, e8524 (2010).

6. Cushion, M. T. \& Arnold, J. Proposal for a Pneumocystis genome project. J. Eukaryot. Microbiol. 44, 7S (1997).

7. Slaven, B. E. et al. Draft assembly and annotation of the Pneumocystis carinii genome. J. Eukaryot. Microbiol. 53(Suppl 1): S89-S91 (2006).

8. Cisse, O. H., Pagni, M. \& Hauser, P. M. De novo assembly of the Pneumocystis jirovecii genome from a single bronchoalveolar lavage fluid specimen from a patient. MBio 4, e00428-00412 (2012).

9. Almeida, J. M., Cisse, O. H., Fonseca, A., Pagni, M. \& Hauser, P. M. Comparative genomics suggests primary homothallism of Pneumocystis species. MBio 6, e02250-02214 (2015).

10. Cisse, O. H., Pagni, M. \& Hauser, P. M. Comparative genomics suggests that the human pathogenic fungus Pneumocystis jirovecii acquired obligate biotrophy through gene loss. Genome Biol. Evol. 6, 1938-1948 (2014).

11. Cushion, M. T. et al. Transcriptome of Pneumocystis carinii during fulminate infection: carbohydrate metabolism and the concept of a compatible parasite. PLOS ONE 2, e423 (2007).

12. Hauser, P. M. et al. Comparative genomics suggests that the fungal pathogen Pneumocystis is an obligate parasite scavenging amino acids from its host's lungs. PLoS ONE 5, e15152 (2010).

13. Porollo, A., Sesterhenn, T. M., Collins, M. S., Welge, J. A. \& Cushion, M. T. Comparative genomics of Pneumocystis species suggests the absence of genes for myo-inositol synthesis and reliance on inositol transport and metabolism. MBio 5, e01834-01814 (2014).

14. Hong, S. T. et al. Pneumocystis carinii karyotypes. J. Clin. Microbiol. 28, 1785-1795 (1990).

15. Stringer, J. R. et al. Molecular genetic distinction of Pneumocystis carinii from rats and humans. J. Eukaryot. Microbiol. 40, 733-741 (1993).

16. Moran, G. P., Coleman, D. C. \& Sullivan, D. J. Comparative genomics and the evolution of pathogenicity in human pathogenic fungi. Eukaryot. Cell 10, 34-42 (2011).

17. Rhind, N. et al. Comparative functional genomics of the fission yeasts. Science 332, 930-936 (2011).
18. Cisse, O. H. et al. Genome sequencing of the plant pathogen Taphrina deformans, the causal agent of peach leaf curl. MBio 4, e00055-00013 (2013).

19. Seoighe, C. et al. Prevalence of small inversions in yeast gene order evolution. Proc. Natl Acad. Sci. USA 97, 14433-14437 (2000).

20. Vestereng, V. H. et al. Quantitative real-time polymerase chain-reaction assay allows characterization of Pneumocystis infection in immunocompetent mice. J. Infect. Dis. 189, 1540-1544 (2004).

21. Katinka, M. D. et al. Genome sequence and gene compaction of the eukaryote parasite Encephalitozoon cuniculi. Nature 414, 450-453 (2001).

22. Ma, L. et al. Sequencing and characterization of the complete mitochondrial genomes of three Pneumocystis species provide new insights into divergence between human and rodent Pneumocystis. FASEB J. 27, 1962-1972 (2013).

23. Deitsch, K. W., Lukehart, S. A. \& Stringer, J. R. Common strategies for antigenic variation by bacterial, fungal and protozoan pathogens. Nat. Rev. Microbiol. 7, 493-503 (2009).

24. Angus, C. W., Tu, A., Vogel, P., Qin, M. \& Kovacs, J. A. Expression of variants of the major surface glycoprotein of Pneumocystis carinii. J. Exp. Med. 183, 1229-1234 (1996).

25. Kutty, G., Maldarelli, F., Achaz, G. \& Kovacs, J. A. Variation in the major surface glycoprotein genes in Pneumocystis jirovecii. J. Infect. Dis. 198, 741-749 (2008)

26. Bishop, L. R., Helman, D. \& Kovacs, J. A. Discordant antibody and cellular responses to Pneumocystis major surface glycoprotein variants in mice. $B M C$ Immunol. 13, 39 (2012).

27. Kutty, G. \& Kovacs, J. A. A single-copy gene encodes Kex1, a serine endoprotease of Pneumocystis jiroveci. Infect. Immun. 71, 571-574 (2003).

28. Lee, L. H. et al. Molecular characterization of KEX1, a kexin-like protease in mouse Pneumocystis carinii. Gene 242, 141-150 (2000).

29. Lugli, E. B., Bampton, E. T., Ferguson, D. J. \& Wakefield, A. E. Cell surface protease PRT1 identified in the fungal pathogen Pneumocystis carinii. Mol. Microbiol. 31, 1723-1733 (1999).

30. Lipschik, G. Y., Masur, H. \& Kovacs, J. A. Polyamine metabolism in Pneumocystis carinii. J. Infect. Dis. 163, 1121-1127 (1991).

31. Furlong, S. T. et al. Lipid transfer from human epithelial cells to Pneumocystis carinii in vitro. J. Infect. Dis. 175, 661-668 (1997).

32. Kornitzer, D. Fungal mechanisms for host iron acquisition. Curr. Opin. Microbiol. 12, 377-383 (2009).

33. Kuznets, G. et al. A relay network of extracellular haem-binding proteins drives C. albicans iron acquisition from haemoglobin. PLoS. Pathog. 10, e1004407 (2014).

34. Kottom, T. J. \& Limper, A. H. Cell wall assembly by Pneumocystis carinii. Evidence for a unique gsc-1 subunit mediating beta -1,3-glucan deposition. J. Biol. Chem. 275, 40628-40634 (2000).

35. Linke, M. J., Cushion, M. T. \& Walzer, P. D. Properties of the major antigens of rat and human Pneumocystis carinii. Infect. Immun. 57, 1547-1555 (1989).

36. Villegas, L. R., Kottom, T. J. \& Limper, A. H. Chitinases in Pneumocystis carini pneumonia. Med. Microbiol. Immunol. 201, 337-348 (2012).

37. Sanchatjate, S. \& Schekman, R. Chs5/6 complex: a multiprotein complex that interacts with and conveys chitin synthase III from the trans-Golgi network to the cell surface. Mol. Biol. Cell. 17, 4157-4166 (2006).

38. Walker, A. N., Garner, R. E. \& Horst, M. N. Immunocytochemical detection of chitin in Pneumocystis carinii. Infect. Immun. 58, 412-415 (1990).

39. Kutty, G., Davis, A. S., Ma, L., Taubenberger, J. K. \& Kovacs, J. A. Pneumocystis encodes a functional endo-beta-1,3-glucanase that is expressed exclusively in cysts. J. Infect. Dis. 211, 719-728 (2015).

40. Kottom, T. J., Hebrink, D. M., Jenson, P. E., Gudmundsson, G. \& Limper, A. H. Evidence for pro-inflammatory beta-1,6 glucans in the Pneumocystis cell wall Infect. Immun. 83, 2816-2826 (2015).

41. Villegas, L. R., Kottom, T. J. \& Limper, A. H. Characterization of PCEng2, a \{beta\}-1,3-endoglucanase homologue in Pneumocystis carinii with activity in cell wall regulation. Am. J. Respir. Cell Mol. Biol. 43, 192-200 (2010).

42. Rappleye, C. A., Eissenberg, L. G. \& Goldman, W. E. Histoplasma capsulatum alpha-(1,3)-glucan blocks innate immune recognition by the beta-glucan receptor. Proc. Natl Acad. Sci. USA 104, 1366-1370 (2007).

43. Cambi, A. et al. Dendritic cell interaction with Candida albicans critically depends on N-linked mannan. J. Biol. Chem. 283, 20590-20599 (2008).

44. Saijo, S. et al. Dectin-2 recognition of alpha-mannans and induction of Th17 cell differentiation is essential for host defense against Candida albicans. Immunity 32, 681-691 (2010).

45. Subramanian, A. et al. Gene set enrichment analysis: a knowledge-based approach for interpreting genome-wide expression profiles. Proc. Natl Acad. Sci. USA 102, 15545-15550 (2005).

46. Irimia, M., Rukov, J. L., Penny, D. \& Roy, S. W. Functional and evolutionary analysis of alternatively spliced genes is consistent with an early eukaryotic origin of alternative splicing. BMC Evol. Biol. 7, 188 (2007).

47. Goffeau, A. et al. Life with 6000 genes. Science 274, 563-547 (1996).

48. Keeling, P. J. et al. The reduced genome of the parasitic microsporidian Enterocytozoon bieneusi lacks genes for core carbon metabolism. Genome Biol. Evol. 2, 304-309 (2010). 
49. Ye, D., Lee, C. H. \& Queener, S. F. Differential splicing of Pneumocystis carinii f. sp. carinii inosine 5'-monophosphate dehydrogenase pre-mRNA. Gene 263, 151-158 (2001).

50. Kornblihtt, A. R. CTCF: from insulators to alternative splicing regulation. Cell Res. 22, 450-452 (2012).

51. Elleuche, S. \& Poggeler, S. Carbonic anhydrases in fungi. Microbiology. 156, 23-29 (2010).

52. Amarsaikhan, N. \& Templeton, S. P. Co-recognition of beta-glucan and chitin and programming of adaptive immunity to Aspergillus fumigatus. Front. Microbiol. 6, 344 (2015).

53. Ma, L. et al. Analysis of variation in tandem repeats in the intron of the major surface glycoprotein expression site of the human form of Pneumocystis carinii. J. Infect. Dis. 186, 1647-1654 (2002).

54. Larsen, H. H. et al. Development of a rapid real-time PCR assay for quantitation of Pneumocystis carinii f. sp. carinii. J. Clin. Microbiol. 40, 2989-2993 (2002).

55. Larsen, H. H. et al. Development and evaluation of a quantitative, touch-down, real-time PCR assay for diagnosing Pneumocystis carinii pneumonia. J. Clin. Microbiol. 40, 490-494 (2002).

56. Morse, C. G. et al. HIV infection and antiretroviral therapy have divergent effects on mitochondria in adipose tissue. J. Infect. Dis. 205, 1778-1787 (2012).

57. Maccallum, I. et al. ALLPATHS 2: small genomes assembled accurately and with high continuity from short paired reads. Genome Biol. 10, R103 (2009).

58. Keely, S. P. et al. Gene arrays at Pneumocystis carinii telomeres. Genetics 170, 1589-1600 (2005).

59. Melnikov, A. et al. Hybrid selection for sequencing pathogen genomes from clinical samples. Genome Biol. 12, R73 (2011).

60. Kovacs, J. A. et al. Identification of antigens and antibodies specific for Pneumocystis carinii. J. Immunol. 140, 2023-2031 (1988).

61. Lundgren, B., Cotton, R., Lundgren, J. D., Edman, J. C. \& Kovacs, J. A. Identification of Pneumocystis carinii chromosomes and mapping of five genes. Infect. Immun. 58, 1705-1710 (1990).

62. Levin, J. Z. et al. Comprehensive comparative analysis of strand-specific RNA sequencing methods. Nat. Methods 7, 709-715 (2010).

63. Parkhomchuk, D. et al. Transcriptome analysis by strand-specific sequencing of complementary DNA. Nucleic Acids Res. 37, e123 (2009).

64. Li, H. et al. The sequence alignment/map format and SAMtools. Bioinformatics 25, 2078-2079 (2009).

65. Langmead, B., Trapnell, C., Pop, M. \& Salzberg, S. L. Ultrafast and memoryefficient alignment of short DNA sequences to the human genome. Genome Biol. 10, R25 (2009).

66. Li, B. \& Dewey, C. N. RSEM: accurate transcript quantification from RNA-Seq data with or without a reference genome. BMC Bioinformatics 12, 323 (2011).

67. Haas, B. J., Zeng, Q., Pearson, M. D., Cuomo, C. A. \& Wortman, J. R. Approaches to fungal genome annotation. Mycology 2, 118-141 (2011).

68. Li, H. Aligning sequence reads, clone sequences and assembly contigs with BWA-MEM. Preprint at http://arxiv.org/abs/1303.3997 (2013).

69. McKenna, A. et al. The Genome Analysis Toolkit: a MapReduce framework for analyzing next-generation DNA sequencing data. Genome Res. 20, 1297-1303 (2010).

70. Watanabe, T. et al. The roles of the C-terminal domain and type III domains of chitinase A1 from Bacillus circulans WL-12 in chitin degradation. J. Bacteriol. 176, 4465-4472 (1994).

71. Rapaka, R. R. et al. Enhanced defense against Pneumocystis carinii mediated by a novel dectin-1 receptor Fc fusion protein. J. Immunol. 178, 3702-3712 (2007).

72. Lionakis, M. S. et al. CX3CR1-dependent renal macrophage survival promotes Candida control and host survival. J. Clin. Invest. 123, 5035-5051 (2013).

73. Anumula, K. R. \& Taylor, P. B. A comprehensive procedure for preparation of partially methylated alditol acetates from glycoprotein carbohydrates. Anal. Biochem. 203, 101-108 (1992).

74. Linke, M. J., Sunkin, S. M., Andrews, R. P., Stringer, J. R. \& Walzer, P. D. Expression, structure, and location of epitopes of the major surface glycoprotein of Pneumocystis carinii f. sp. carinii. Clin. Diagn. Lab. Immunol. 5, 50-57 (1998).

75. Yin, X. et al. Glycoproteomic analysis of the secretome of human endothelial cells. Mol. Cell. Proteomics 12, 956-978 (2013).

\section{Acknowledgements}

This project has been funded in whole or in part with federal funds from the Intramural Research Program of the US National Institutes of Health Clinical Center; the National
Institute of Allergy and Infectious Diseases; the National Cancer Institute, the National Institutes of Health, under Contract No. HHSN261200800001E; the National Human Genome Research Institute (grant U54HG003067 to the Broad Institute); and the National Institutes of Health (NIH/NCRR)-funded grant entitled 'Integrated Technology Resource for Biomedical Glycomics' (grant 1 P41 RR018502-01) to the Complex Carbohydrate Research Center. Y.X. was supported by a scholarship from the Chongqing Medical University (Chongqing, China). X.D was supported by the Public Health Bureau of Guangzhou City (grant 2009-YB-089009) and the Department of Public Health, Guangdong Province (grant 2012513), China. X.S. was supported by the China Scholarship Council. We thank Rene Costello and Howard Mostowski for their support of the animal studies; Michail Lionakis for providing Candida-infected mouse tissue; Chad Steele for providing the dectin-Fc construct; Peter Walzer and Michael Linke for providing antibody RA-E7; the Broad Institute Genomics Platform for Illumina sequencing of DNA and RNA samples; Carsten Russ and Sinéad Chapman for coordination of Illumina sequencing at the Broad Institute; Jennifer Troyer for coordination of Pacific Bioscience sequencing at Leidos Inc.; Zuoming Deng and Biswajit Das for help with genome assembly; Alexander Li and Lily Lin for assistance with rDNA PCR; Jing Zhang for assistance with DNA hybridization; Margaret Priest for assistance with annotation; Lucia Alvarado-Balderrama for assistance with genome data submission and release; Rhys Farrer for assistance with displaying synteny; and Henry Masur, June Kwon-Chung, Peter Williamson, John Bennett and Christopher Desjardins for reviewing the manuscript.

\section{Author contributions}

L.M., Z.C., D.W.H., R.S., C.N., B.W.B., P.A., R.A.L., C.A.C. and J.A.K. were involved in study design. L.M., G.K., L.B., G.F., E.G. and M.S. were involved in sample preparation. A.A., M.F., J.M.G., P.M., A.M., S.Y. and Q.Z. were involved in Illumina sequencing. L.F. and J.Z.L. were involved in Illumina RNA sequencing. D.W.H., X.H., X.J., B.T.S., J.Y. and X.Z were involved in 454 sequencing. D.W.H., K.J., C.R., B.T. and R.S. were involved in PacBio sequencing. E.D., R.D., X.D., E.G., G.H., Y.L., X.S., L.W. and Y.X. were involved in PCR and Sanger sequencing. C.H., X.D., Y.X. and X.S. performed Southern blot. A.A., S.S., S.Y., P.M., M.F., J.M.G., Q.Z., Z.C., D.W.H., X.H., B.T.S. and X.Z. were involved in genome assembly and annotation. Z.C., C.A.C., L.M. and J.A.K. were involved in comparative genomic analysis. G.K., M.I. and P.A. were involved in chitin analysis. G.K., M.I., H.W. and P.A. were involved in msg and glycan MS. L.M., Z.C., C.A.C. and J.A.K. were involved in manuscript writing. All authors reviewed the manuscript. The content of this publication does not necessarily reflect the views or policies of the Department of Health and Human Services, nor does mention of trade names, commercial products or organizations imply endorsement by the US Government.

\section{Additional information}

Accession codes: Illumina raw reads have been deposited in the NCBI Sequence Read Archive for the genome sequencing of $P$. murina (accession codes SRX248091 and SRX1435036), P. carinii (accession codes SRX387635 and SRX387636) and P. jirovecii (accession code SRX387638). Illumina raw reads for RNA-Seq of $P$. murina and $P$. carinii have been deposited in the NCBI Sequence Read Archive under accession code PRJNA292577. Genome assemblies and annotations have been deposited in the NCBI BioProject database with accession codes PRJNA70803 (P. murina), PRJNA223511 (P. carinii) and PRJNA223510 (P. jirovecii). All sequence data has been linked to NCBI Umbrella project PRJNA223519.

Supplementary Information accompanies this paper at http://www.nature.com/ naturecommunications

Competing financial interests: The authors declare no competing financial interests.

Reprints and permission information is available online at http://npg.nature.com/ reprintsandpermissions/

How to cite this article: Ma, L. et al. Genome analysis of three Pneumocystis species reveals adaptation mechanisms to life exclusively in mammalian hosts. Nat. Commun. 7:10740 doi: 10.1038/ncomms10740 (2016).

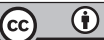

This work is licensed under a Creative Commons Attribution 4.0 International License. The images or other third party material in this article are included in the article's Creative Commons license, unless indicated otherwise in the credit line; if the material is not included under the Creative Commons license, users will need to obtain permission from the license holder to reproduce the material. To view a copy of this license, visit http://creativecommons.org/licenses/by/4.0/ 